TRANSACTIONS OF THE

AMERICAN MATHEMATICAL SOCIETY

Volume 356, Number 6, Pages 2251-2279

S 0002-9947(03)03480-9

Article electronically published on October 8, 2003

\title{
MAXIMUM NORMS OF RANDOM SUMS AND TRANSIENT PATTERN FORMATION
}

\author{
THOMAS WANNER
}

\begin{abstract}
Many interesting and complicated patterns in the applied sciences are formed through transient pattern formation processes. In this paper we concentrate on the phenomenon of spinodal decomposition in metal alloys as described by the Cahn-Hilliard equation. This model depends on a small parameter, and one is generally interested in establishing sharp lower bounds on the amplitudes of the patterns as the parameter approaches zero. Recent results on spinodal decomposition have produced such lower bounds. Unfortunately, for higher-dimensional base domains these bounds are orders of magnitude smaller than what one would expect from simulations and experiments. The bounds exhibit a dependence on the dimension of the domain, which from a theoretical point of view seemed unavoidable, but which could not be observed in practice.

In this paper we resolve this apparent paradox. By employing probabilistic methods, we can improve the lower bounds for certain domains and remove the dimension dependence. We thereby obtain optimal results which close the gap between analytical methods and numerical observations, and provide more insight into the nature of the decomposition process. We also indicate how our results can be adapted to other situations.
\end{abstract}

\section{INTRODUCTION}

Many physical and biological processes exhibit pattern formation mechanisms which lead to intriguing and complicated patterns. In many cases, these patterns are transient and contain an apparent stochastic element. One example of such a mechanism can be observed in metal alloys and is called spinodal decomposition [10. The underlying physics can be described as follows. If a homogeneous high-temperature mixture of two metallic components is rapidly quenched below a certain temperature, a sudden phase separation may set in, during which the mixture quickly becomes inhomogeneous and forms a fine-grained structure, more or less alternating between the two alloy components. If the experiment is repeated under almost identical conditions, the fine-structure of the observed pattern will be significantly different, even though the overall characteristics remain unchanged and one would immediately label the new pattern as "similar" to the previous one. Common to the generated patterns is a geometry as shown in Figure 1, which exhibits a characteristic thickness of the snake-like structures.

Received by the editors September 3, 2002.

2000 Mathematics Subject Classification. Primary 35K35, 35B05, 42A61.

Key words and phrases. Spinodal decomposition, Cahn-Hilliard equation, pattern formation, probabilistic aspects, random sums of functions. 
In order to describe this and other phase separation processes in binary metal alloys, Cahn and Hilliard [8, 11] proposed the fourth-order parabolic partial differential equation

$$
\begin{array}{r}
u_{t}=-\Delta\left(\varepsilon^{2} \Delta u+f(u)\right) \quad \text { in } \quad \Omega, \\
\frac{\partial u}{\partial \nu}=\frac{\partial \Delta u}{\partial \nu}=0 \quad \text { on } \quad \partial \Omega,
\end{array}
$$

where $\Omega \subset \mathbb{R}^{n}$ is a suitable bounded domain with $n \in\{1,2,3\}$, and the function $f$ is the negative of the derivative of a double-well potential $F$. Usually one considers the specific potential $F(u)=\left(u^{2}-1\right)^{2} / 4$ which leads to the cubic function $f(u)=u-u^{3}$. Furthermore, $\varepsilon$ is a small positive parameter modeling interaction length. In this formulation, the variable $u$ represents the concentration of one of the two components of the alloy, subject to an affine transformation such that the concentrations 0 or 1 correspond to $u$ being -1 or 1 , respectively. The Cahn-Hilliard equation is mass-conserving in the sense that the total concentration $\int_{\Omega} u(t, x) d x$ remains constant along any solution $u$. In addition, (11) is a gradient system with respect to the standard van der Waals free energy functional; see for example Fife 20]. For more details on spinodal decomposition and how it is modeled by the Cahn-Hilliard equation we refer the reader to Cahn [9, 10, Elder, Desai [15], Elder, Rogers, Desai [16], Hilliard [24], Hyde et al. [25], and Langer [27]. Numerical simulations can be found in Bai et al. [3, 4, Copetti [13, Copetti, Elliott [14], Elliott [17], Elliott, French [18], Hyde et al. [25], Nash [32], and Sander, Wanner [33].

It is clear from the form of the equation that every constant function $\bar{u}_{o} \equiv \mu$ is an equilibrium solution of (1). This equilibrium is unstable if $\mu$ is contained in the spinodal interval, which consists of the usually connected set of all $\mu \in \mathbb{R}$ for which $f^{\prime}(\mu)>0$. Thus, if $\mu$ lies in the spinodal interval, any orbit of (1) originating at an initial condition $u_{o} \approx \bar{u}_{o}$ is likely to be driven away from $\bar{u}_{o}$. In order to explain

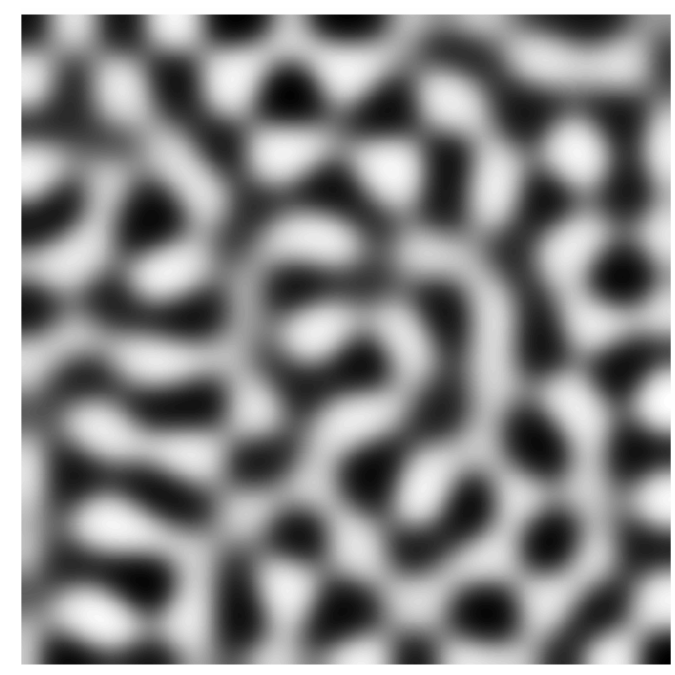

FiguRE 1. The geometry of a typical pattern observed during spinodal decomposition in the Cahn-Hilliard equation, where $\Omega=$ $(0,1)^{2}$ and $\varepsilon=0.015$. 
spinodal decomposition, one has to understand exactly how such solutions depart from the homogeneous equilibrium.

A natural first step is to study the linearization of (11) at the homogeneous equilibrium $\bar{u}_{o} \equiv \mu$, which is given by

$$
\begin{array}{ccc}
v_{t}=-\Delta\left(\varepsilon^{2} \Delta v+f^{\prime}(\mu) v\right)=: A_{\varepsilon} v & \text { in } & \Omega, \\
\frac{\partial v}{\partial \nu}=\frac{\partial \Delta v}{\partial \nu}=0 & \text { on } & \partial \Omega .
\end{array}
$$

If we introduce the Hilbert space

$$
X=\left\{v \in L^{2}(\Omega): \int_{\Omega} v d x=0\right\}
$$

then the operator $A_{\varepsilon}: X \rightarrow X$ defined in (2), associated with the domain

$$
D\left(A_{\varepsilon}\right)=\left\{v \in X \cap H^{4}(\Omega): \frac{\partial v}{\partial \nu}(x)=\frac{\partial \Delta v}{\partial \nu}(x)=0, x \in \partial \Omega\right\},
$$

is self-adjoint, and $-A_{\varepsilon}$ is sectorial. Let $0<\kappa_{1} \leq \kappa_{2} \leq \ldots \rightarrow \infty$ denote the eigenvalues of the negative Laplacian $-\Delta: X \rightarrow X$ subject to homogeneous Neumann boundary conditions, and denote the corresponding complete set of $L^{2}(\Omega)$ orthonormalized eigenfunctions by $\psi_{1}, \psi_{2}, \ldots$. Then the spectrum of $A_{\varepsilon}$ consists of the eigenvalues

$$
\lambda_{k, \varepsilon}=\kappa_{k} \cdot\left(f^{\prime}(\mu)-\varepsilon^{2} \kappa_{k}\right), \quad k \in \mathbb{N},
$$

with corresponding eigenfunctions $\psi_{k}$. These eigenvalues are bounded above by

$$
\lambda_{\varepsilon}^{\max }=\frac{f^{\prime}(\mu)^{2}}{4 \varepsilon^{2}}
$$

and asymptotically as $\varepsilon \rightarrow 0$, the largest eigenvalue grows like $\lambda_{\varepsilon}^{\max }$.

The dynamics of (2) can roughly be described as follows. The strongest unstable directions are the ones corresponding to $\kappa_{k} \approx f^{\prime}(\mu) /\left(2 \varepsilon^{2}\right)$, and one would expect that most solutions of (2) originating near the (unique) equilibrium 0 are driven away in the direction of some of these eigenfunctions.

Mathematical results for the nonlinear Cahn-Hilliard equation (1) have been obtained by Grant 222, Maier-Paape, Wanner [28, 29], and Sander, Wanner [34]. All of these studies use information about the linearization (2) and relate it to the evolution of solutions of (1) originating near $\bar{u}_{o}$. This is achieved by employing a dynamical systems approach in the sense that they consider (10) as an abstract evolution equation on a suitable function space. More precisely, equation (1) generates a nonlinear semiflow $T_{\varepsilon}(t), t \geq 0$, on the affine space $\mu+X^{1 / 2}$, where $X^{1 / 2}$ denotes the Hilbert space

$$
X^{1 / 2}=\left\{v \in H^{2}(\Omega) \cap X: \frac{\partial v}{\partial \nu}=0 \text { on } \partial \Omega\right\} .
$$

The constant function $\bar{u}_{o} \equiv \mu$ is an equilibrium point for $T_{\varepsilon}$, and the linearization of $T_{\varepsilon}$ at $\bar{u}_{o}$ is given by the analytic semigroup $S_{\varepsilon}$ generated by $A_{\varepsilon}$.

Using the above setting, the results of [22, 28, 29, 34] can be described as follows. The first explanation of spinodal decomposition in the Cahn-Hilliard equation was obtained by Grant [22], who considered the case of one-dimensional domains $\Omega$. His approach, however, failed to explain the complicated patterns observed in higher space dimensions. For more details see the introduction of [28]. 
The case of higher-dimensional domains was first addressed by Maier-Paape, Wanner [28, 29]. They obtained a description of the patterns, an estimate for the characteristic thickness of the patterns, as well as a dynamical description of the early decomposition process. The latter result was achieved by relating the dynamics of (1) in a neighborhood $V_{\varepsilon}$ of the equilibrium $\bar{u}_{o}$ to the dynamics of the linearized equation (2). Their results state that most solutions of (1) starting in a smaller neighborhood $U_{\varepsilon} \subset V_{\varepsilon}$ of $\bar{u}_{o}$ exit $V_{\varepsilon}$ close to the linear subspace

$$
X_{\varepsilon}^{+}=\operatorname{span}\left\{\psi_{k}: \lambda_{k, \varepsilon} \geq \gamma_{o} \cdot \lambda_{\varepsilon}^{\max }\right\} \subset X^{1 / 2},
$$

where $0 \ll \gamma_{o}<1$. This dominating subspace is spanned by the eigenfunctions corresponding to a small percentage of the largest eigenvalues of $A_{\varepsilon}$, its dimension is proportional to $\varepsilon^{-\operatorname{dim} \Omega}$, and functions in $X_{\varepsilon}^{+}$generally exhibit patterns similar to the one depicted in Figure 1 .

The results by Maier-Paape and Wanner successfully describe both the variety and the common characteristics of all patterns observed during spinodal decomposition. The variety of the patterns is due to the fact that different initial conditions $u_{o} \in U_{\varepsilon}$ lead to different exit points from $V_{\varepsilon}$. On the other hand, most of these exit points are close to the finite-dimensional subspace $X_{\varepsilon}^{+}$, which is responsible for the common characteristics of the patterns.

Nevertheless, these results are far from optimal. In order for the theory of [28, 29] to hold, the size of the neighborhood $V_{\varepsilon}$ has to be proportional to $\varepsilon^{\operatorname{dim} \Omega}$ with respect to the $H^{2}(\Omega)$-norm. This is in stark contrast to the numerical simulations for one-dimensional domains performed by Sander, Wanner [33. Their simulations indicate that the predicted patterns are observed until the maximum norm of the solution reaches an $\varepsilon$-independent threshold, which corresponds to the $H^{2}(\Omega)$-norm being of the order $\varepsilon^{-2}$. For small values of $\varepsilon$ this number is orders of magnitude larger than the admissible size of $V_{\varepsilon}$. In that sense, the results of [28, 29] only describe the early stages of spinodal decomposition. In the neighborhood $V_{\varepsilon}$, spinodal decomposition is mostly a linearly driven phenomenon — outside of this neighborhood the nonlinearity is generally dominant and the results of [28, 29] do not apply.

Based on the numerical insight gained in 33], Sander and Wanner [34] developed a new approach to understanding the subsequent stages of spinodal decomposition. They could identify a region $\mathcal{R}_{\varepsilon}$ in phase space, which extends well beyond the neighborhood $V_{\varepsilon}$, but in which the influence of the nonlinearity remains extremely small. Leaving out technical details, their main result can be stated as follows.

Theorem 1.1 (Theorem 3.4 in [34]). Consider (11) with $f(u)=u-u^{3}$ and $\mu=0$. Let $\varrho>0$ be arbitrarily small, but fixed, and let $u_{o}$ denote an initial condition close to $\bar{u}_{o} \equiv \mu$, which is sufficiently close to the dominating subspace $X_{\varepsilon}^{+}$defined in (8). Finally, let $u$ and $v$ be the solutions to (11) and (2), respectively, with initial condition $u_{o}$. Then as long as

$$
\|u(t)\|_{H^{2}(\Omega)} \leq C \cdot \varepsilon^{-1+\varrho+\operatorname{dim} \Omega / 4} \cdot\left\|u_{o}\right\|_{H^{2}(\Omega)}^{\varrho},
$$

we have

$$
\frac{\|u(t)-v(t)\|_{H^{2}(\Omega)}}{\|v(t)\|_{H^{2}(\Omega)}} \leq C \cdot \varepsilon^{2-\operatorname{dim} \Omega / 2} .
$$

In other words, u remains extremely close to $v$ until $\|u(t)\|_{H^{2}(\Omega)}$ exceeds the threshold given in (9). 

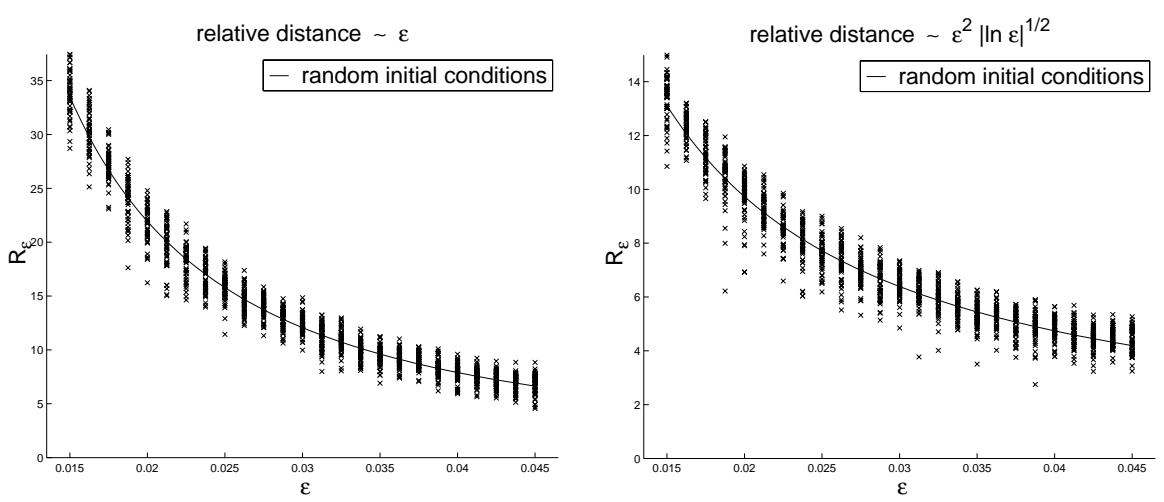

Figure 2. Observed behavior: Simulation results for the CahnHilliard equation on $\Omega=(0,1)^{2}$. For each considered $\varepsilon$, fifty initial conditions are chosen randomly with $H^{2}(\Omega)$-norm $10^{-4}$, and the corresponding solutions $u(t)$ and $v(t)$ of (1) and (2) are computed until the relative distance reaches a certain threshold. The $H^{2}(\Omega)$ norm of $u(t)$ at this point is denoted by $R_{\varepsilon}$. In the left diagram, the relative distance is $\varepsilon / 9$; the best fit for the curve is $R_{\varepsilon} \approx$ $0.0689 \cdot \varepsilon^{-1.4731}$. In the right diagram, the relative distance is 0.56 . $\varepsilon^{2} \cdot \sqrt{|\ln \varepsilon|} ;$ the best fit for the curve is $R_{\varepsilon} \approx 0.1681 \cdot \varepsilon^{-1.0374}$.

Recalling that at the end of the early decomposition stage, most solutions of (1) originating near $\bar{u}_{o}$ exit the neighborhood $V_{\varepsilon}$ close to the dominating subspace $X_{\varepsilon}^{+}$, we see that Theorem 1.1 applies to all of them. It shows that even outside the neighborhood $V_{\varepsilon}$, spinodal decomposition is a linearly driven phenomenon, due to the fact that most solutions are driven into the highly localized region $\mathcal{R}_{\varepsilon}$ of phase space in which the behavior is essentially linear - while outside of $\mathcal{R}_{\varepsilon}$ nonlinear effects clearly dominate. This has been demonstrated in [33].

Theorem 1.1 marks a big step towards explaining the numerical results of [33] in one space dimension, which indicate that the relative distance $\|u-v\|_{H^{2}(\Omega)} /\|v\|_{H^{2}(\Omega)}$ between the nonlinear solution $u$ and the linear solution $v$ remains bounded by some small $\varepsilon$-independent threshold, as long as the norm of the nonlinear solution is bounded by $C \varepsilon^{-2}$. While Theorem 1.1 does not reproduce exponent -2 , it furnishes a much better threshold for the relative distance, namely $O\left(\varepsilon^{2-\operatorname{dim} \Omega / 2}\right)-$ leaving considerable room for improvement. In fact, by considering nonlinearities other than $f(u)=u-u^{3}$, it is possible to improve the above order to $\varepsilon^{-2+\varrho+\operatorname{dim} \Omega / 2}$, for any $\varrho>0$ [34]. This is in good agreement with the one-dimensional simulations.

Physically most interesting are of course the cases of two- and three-dimensional base domains $\Omega$. The results in 28, 29, 34 seem to indicate that as the dimension increases, the radius to which linear behavior can be observed decreases significantly. Notice for example in Theorem 1.1 that both the right-hand side of (9) and the bound on the relative distance in (10) are worse for higher dimensions. Numerical simulations do not confirm this dimension dependence. Nash 32] performed simulations for two-dimensional $\Omega$ which are analogous to the ones in [33], and she obtained the same exponent -2 as in the one-dimensional case. While these simulations do not necessarily imply that Theorem 1.1 is sub-optimal, the left diagram 

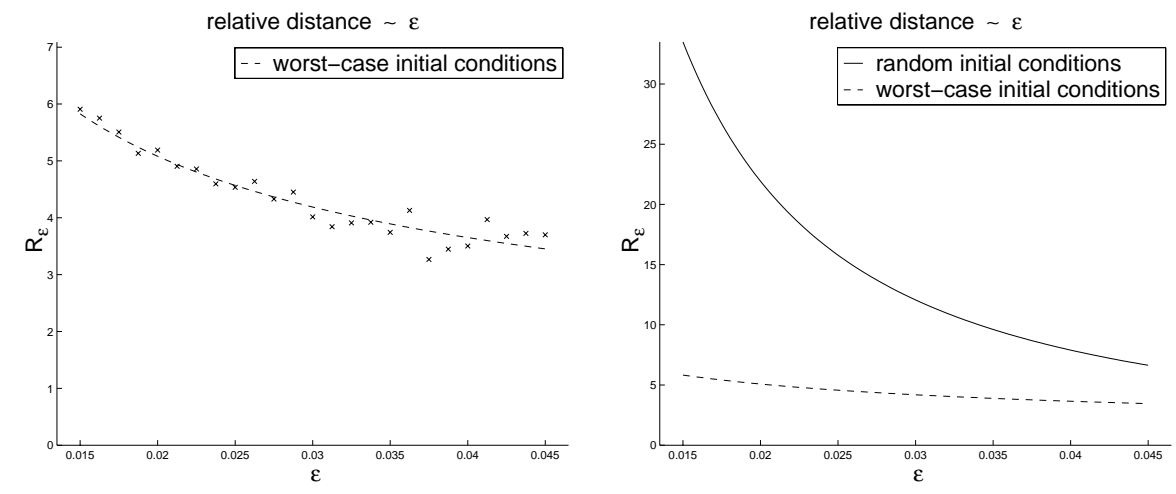

FiguRE 3. Worst-case behavior: Simulation results for the CahnHilliard equation on $\Omega=(0,1)^{2}$. For each value of $\varepsilon$, the initial condition is chosen as in (37), scaled to an $H^{2}(\Omega)$-norm of $10^{-4}$, and the corresponding solutions $u(t)$ and $v(t)$ of (1D) and (2) are computed until the relative distance reaches $\varepsilon / 9$. The $H^{2}(\Omega)$-norm of $u(t)$ at this point is denoted by $R_{\varepsilon}$. The best fit for the curve is $R_{\varepsilon} \approx 0.79 \cdot \varepsilon^{-0.48}$. The results are shown in the left diagram. For comparison, the right diagram contrasts the fitted curves for worst-case and randomly chosen initial conditions.

in Figure 2 does. It shows simulation results for $\Omega=(0,1)^{2}$ and $f(u)=u-u^{3}$ obtained by randomly choosing initial conditions $u_{o}$ close to the homogeneous equilibrium 0 , following the linear and nonlinear solutions originating at $u_{o}$ until their relative distance exceeds $\varepsilon / 9$, and recording the corresponding $H^{2}(\Omega)$-norm of $u(t)$. A least-squares fit of these norms indicates roughly an order of $\varepsilon^{-3 / 2}$, which is considerably larger than the $\varepsilon^{-1 / 2}$ predicted by Theorem 1.1. In fact, the right diagram of Figure 2 shows that even for the smaller relative distance $O\left(\varepsilon^{2} \sqrt{|\ln \varepsilon|}\right)$ the observed order considerably exceeds the predicted one. Similar simulations for larger ranges of $\varepsilon$-values are consistent with the ones presented here.

These simulations seem to indicate that Theorem 1.1 is highly sub-optimal in dimensions two and three, and that the estimates of [34] are not sharp. Yet, the situation is not quite as simple as that. In Section 4.1 below we construct a family of functions $w_{\varepsilon} \in X_{\varepsilon}^{+}$which establishes the optimality of the crucial estimate in the proof of Theorem 1.1. What happens if we perform numerical simulations analogous to the ones leading to Figure 2, but instead of choosing the initial conditions $u_{o}$ randomly for each $\varepsilon$, we consider $u_{o}=w_{\varepsilon}$ ? The results of these simulations are shown in Figure 3, and they indicate that for these functions Theorem 1.1 is optimal in two space dimensions.

The above discussion shows that there is no hope of universally improving the results in [34]. Yet, a closer look at the shape of the functions $w_{\varepsilon}$ constructed in Section 4.1 reveals that the situation is more delicate. Figures 4 and 5 depict $w_{\varepsilon}$ in the one- and two-dimensional situations, for two different $\varepsilon$-values each. While these functions are contained in the dominating subspace $X_{\varepsilon}^{+}$, their patterns do not resemble the ones normally observed during spinodal decomposition. The functions $w_{\varepsilon}$ exhibit a very localized structure, which is not shared by the majority of 

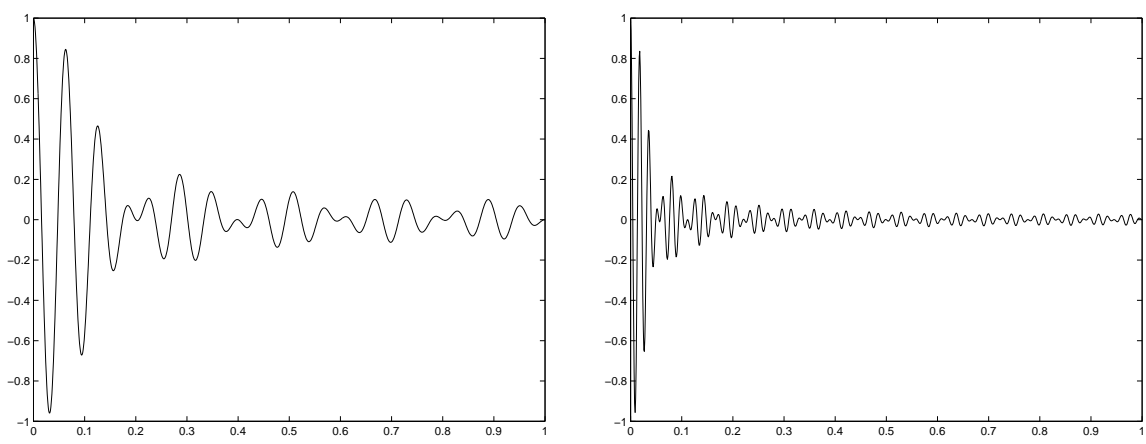

Figure 4. One-dimensional examples which realize the worstcase estimate. Each function is contained in the dominating subspace $X_{\varepsilon}^{+}$for $\gamma_{o}=0.9$. In the left diagram we have $\varepsilon=0.007$ and $\operatorname{dim} X_{\varepsilon}^{+}=10$; the right diagram is for $\varepsilon=0.002$ and $\operatorname{dim} X_{\varepsilon}^{+}=36$.
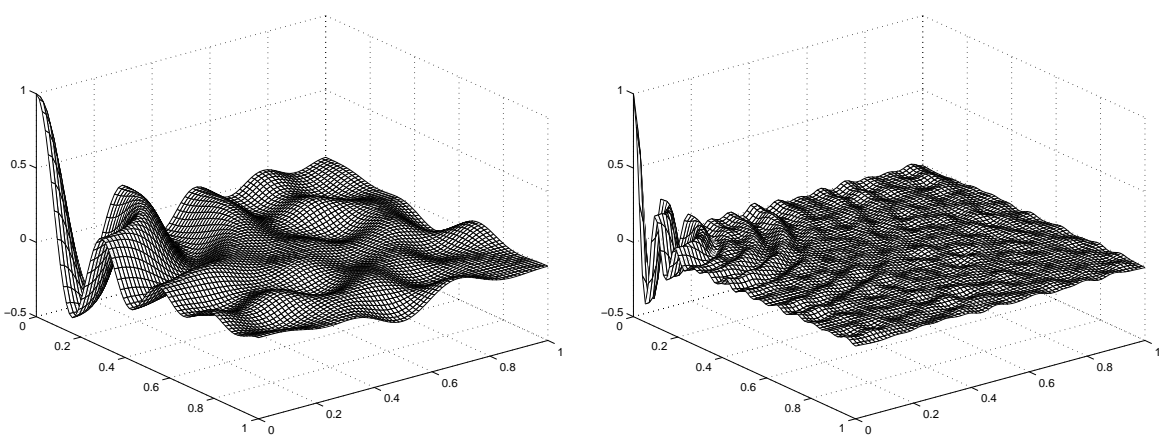

Figure 5. Two-dimensional examples which realize the worstcase estimate. Each function is contained in the dominating subspace $X_{\varepsilon}^{+}$for $\gamma_{o}=0.9$. In the left diagram we have $\varepsilon=0.03$ and $\operatorname{dim} X_{\varepsilon}^{+}=32$; the right diagram is for $\varepsilon=0.01$ and $\operatorname{dim} X_{\varepsilon}^{+}=257$.

functions in or close to $X_{\varepsilon}^{+}$. It turns out that the worst-case behavior predicted by Theorem 1.1 is only realized by a small set of initial conditions $u_{o}$, while for most $u_{o}$ linear behavior can be observed to much larger distances, as indicated in Figure 2,

In this paper, we confirm this expectation. Using probabilistic methods on the maximum norms of random sums, we are able to prove that the set of initial conditions for which Theorem 1.1 is sharp is small in a measure-theoretic sense. For most initial conditions, we obtain considerably better results. Leaving out technical details, our main result can be stated as follows. The detailed version of this result can be found in Section 4.2 see Theorem 4.7

Theorem 1.2. Consider (11) with $f(u)=u-u^{3}$ and $\mu=0$, and let $\Omega$ be a bounded rectangular domain in $\mathbb{R}^{n}$, where $n \in\{1,2,3\}$. Let $\varrho>0$ be arbitrarily small, but fixed, and let $u_{o}$ denote an initial condition close to $\bar{u}_{o} \equiv \mu$, which is sufficiently close to the dominating subspace $X_{\varepsilon}^{+}$defined in (8). Finally, let $u$ and $v$ be the solutions to (11) and (2), respectively, starting at $u_{o}$. Then for most such initial 
conditions $u_{o}$, as long as

$$
\|u(t)\|_{H^{2}(\Omega)} \leq C \cdot \varepsilon^{-1+\varrho} \cdot\left\|u_{o}\right\|_{H^{2}(\Omega)}^{\varrho},
$$

we have

$$
\frac{\|u(t)-v(t)\|_{H^{2}(\Omega)}}{\|v(t)\|_{H^{2}(\Omega)}} \leq C \cdot \varepsilon^{2} \cdot \sqrt{|\ln \varepsilon|} .
$$

The above result considerably improves Theorem 1.1 in dimensions two and three. Notice that (11) and (12) are independent of the dimension of $\Omega$. The radius in (9) has been increased by a factor of $\varepsilon^{-\operatorname{dim} \Omega / 4}$, while at the same time the relative distance bound has been improved by a factor of $\varepsilon^{\operatorname{dim} \Omega / 2} \sqrt{|\ln \varepsilon|}$. Furthermore, the right diagram of Figure 2 indicates that Theorem 1.2 is optimal for $\Omega=(0,1)^{2}$.

Similar to [34, we can improve the order in (11) by considering nonlinearities of the form $f_{\sigma}(u)=u-u^{1+\sigma}$, where $\sigma \geq 1$. The corresponding double-well potentials $F_{\sigma}$ are given by $F_{\sigma}(u)=u^{2+\sigma} /(2+\sigma)-u^{2} / 2$, which for $\sigma \rightarrow \infty$ approach the nonsmooth free energy discussed by Blowey and Elliott [6, 7]. We show in Section 4.2 that for $\mu=0$ and a nonlinearity of this form, the radius to which linear behavior can be observed is larger: The right-hand side of (11) can be replaced by

$$
C \cdot \varepsilon^{-2 \cdot(1-1 / \sigma)+\varrho} \cdot\left\|u_{o}\right\|_{H^{2}(\Omega)}^{\varrho} .
$$

Thus, by choosing a suitable double-well potential $F_{\sigma}$, we can get as close to the order estimate $\varepsilon^{-2}$ as we wish. Combined with [29, Remark 3.6] this establishes spinodal decomposition for these potentials until the $L^{\infty}(\Omega)$-norm reaches an almost $\varepsilon$-independent threshold - thereby describing the complete spinodal decomposition process for the first time.

The remainder of this paper is organized as follows. In the next section we describe briefly the functional analytic setting and our standing assumptions. In Section 3 we derive sharp probabilistic bounds on the maximum norms of random sums. These results are inspired by previous work of Aurich et al. 2] and Kahane [26]. In contrast to their situations, however, we have to consider oneparameter families of random sums. These results are then used to obtain sharp dimension-independent bounds for the nonlinearity of the Cahn-Hilliard equation in Section 4.1. The estimates are realized with high probability, and enable us to prove our main results in Section 4.2. Finally, in Section 5 we point to further possible improvements of our results. We finish by indicating generalizations to other situations, such as the Turing mechanism in parabolic systems and Cahn-Hilliard models involving elasticity. Throughout the paper, capitalized constants $C, C_{1}, C_{2}, \ldots$ refer to generic $\varepsilon$-independent positive constants, whose value might change from line to line.

\section{Basic ASSUmptions AND ABStRaCt SETting}

In this brief section we state our standing assumptions, present the functionalanalytic setting for our studies, and recall some known results for the linearized Cahn-Hilliard equation (2), which will be needed in the following. Finally, we state some properties of the dominating subspace defined in (8).

2.1. Assumptions and functional-analytic setting. We consider the nonlinear Cahn-Hilliard equation as defined in (1), and assume that the following hold:

(A1) Let $\Omega \subset \mathbb{R}^{n}$ be a rectangular bounded domain, where $n \in\{1,2,3\}$. 
(A2) Let $f: \mathbb{R} \rightarrow \mathbb{R}$ be a $C^{4}$-function, and assume that the mass $\mu \in \mathbb{R}$ is chosen in such a way that $f^{\prime}(\mu)>0$.

In order to simplify the presentation, we follow 29, 34 and perform a change of variables so that the mass constraint $\int_{\Omega} u d x=\mu$ can be replaced by the mass constraint $\int_{\Omega} u d x=0$. This leads to the transformed equation

$$
\begin{gathered}
u_{t}=-\Delta\left(\varepsilon^{2} \Delta u+f(\mu+u)\right) \quad \text { in } \quad \Omega, \\
\frac{\partial u}{\partial \nu}=\frac{\partial \Delta u}{\partial \nu}=0 \quad \text { on } \quad \partial \Omega, \\
\int_{\Omega} u d x=0 .
\end{gathered}
$$

It is clear that if $u$ is any solution of (13), then the sum $\mu+u$ solves the original Cahn-Hilliard equation (1), and vice versa. It was shown in 29] that (13) can be viewed as an abstract evolution equation of the form

$$
u_{t}=A_{\varepsilon} u+F(u) .
$$

The linear operator $A_{\varepsilon}$ is defined as in (2), and the nonlinearity $F$ is given by

$$
F(u)=-\Delta g(u) \quad \text { where } \quad g(u)=f(\mu+u)-f^{\prime}(\mu) u-f(\mu) .
$$

In order to derive sharp nonlinearity estimates for $F$ in Section 4.1 we need more precise knowledge of the local behavior of $g$.

(A3) Assume that (A2) holds and let $g$ be defined as in (15). We assume that $g$ satisfies $g(u)=u^{1+\sigma} \cdot \tilde{g}(u)$, where $\sigma \geq 1$ and $\tilde{g}$ is $C^{2}$ on an open interval containing 0 .

For the standard Cahn-Hilliard equation with $\mu=0$ and $f(u)=u-u^{3}$ this condition is satisfied with $\sigma=2$, for the case $\mu \neq 0$ we have $\sigma=1$. Notice that in the latter case we have to consider the transformed equation (13).

The functional-analytic setting for the abstract evolution equation (14) can be described as follows. Let $X$ be defined as in (3). Then the operator $A_{\varepsilon}: X \rightarrow X$ with domain (4) is self-adjoint, and $-A_{\varepsilon}$ is sectorial. Thus, we can define the fractional power spaces $X^{1 / 2, \varepsilon} \subset X$ equipped with the operator norm $\|\cdot\|_{1 / 2, \varepsilon}$; see for example Henry [23. These spaces are Hilbert spaces, and at least in principle could depend on the parameter $\varepsilon$. However, it was shown in 29 that these spaces are independent of $\varepsilon$ and coincide with the space $X^{1 / 2}$ defined in (7). While the norms $\|\cdot\|_{1 / 2, \varepsilon}$ do depend on $\varepsilon$, they are all equivalent to the standard $H^{2}(\Omega)$-norm on $X^{1 / 2}$. For our applications, it is more convenient to work with the nonstandard norm

$$
\|u\|_{*}=\sqrt{\|u\|_{L^{2}(\Omega)}^{2}+\|\Delta u\|_{L^{2}(\Omega)}^{2}} \quad \text { for } \quad u \in X^{1 / 2} .
$$

Assumption (A1) guarantees that this norm is equivalent to the standard $H^{2}(\Omega)$ norm, and therefore also to the operator norms $\|\cdot\|_{1 / 2, \varepsilon}$. Furthermore, (A2) implies that the nonlinearity $F$ defined in (15) is a continuously differentiable nonlinear operator from $X^{1 / 2}$ to $X$, see [22, 29]. The theory of Henry [23] now shows that the abstract evolution equation (14) generates a nonlinear semiflow $T_{\varepsilon}(t), t \geq 0$, on the Hilbert space $X^{1 / 2}$. 
2.2. Properties of the linearization. The basic properties of the linearized Cahn-Hilliard operator $A_{\varepsilon}$ defined in (2) were already mentioned in the Introduction. In particular, they imply that $A_{\varepsilon}$ is the generator of an analytic semigroup $S_{\varepsilon}(t), t \geq 0$, on the Hilbert space $X$. This semigroup has the explicit representation

$$
S_{\varepsilon}(t) v=\sum_{k=1}^{\infty} e^{\lambda_{k, \varepsilon} \cdot t} \cdot\left(v, \psi_{k}\right)_{L^{2}(\Omega)} \cdot \psi_{k} \quad \text { for } \quad v \in X
$$

where $\lambda_{k, \varepsilon}$ was defined in (5), and $\psi_{k}$ denotes the $k$-th eigenfunction of the negative Laplacian on $X$ subject to homogeneous Neumann boundary conditions. (See the Introduction.) Regarding the asymptotic behavior of $S_{\varepsilon}(t)$, we recall the following result from Sander, Wanner [34].

Lemma 2.1. Let $A_{\varepsilon}$ be as in (21), consider the spaces $X$ and $X^{1 / 2}$ defined in (3) and (7), respectively, and let $S_{\varepsilon}(t)$ denote the analytic semigroup generated by $A_{\varepsilon}$. Let $X_{\varepsilon}^{+}$be defined as in (8) for some $\gamma_{o} \in(0,1)$, and let $X_{\varepsilon}^{-}$denote its orthogonal complement in $X^{1 / 2}$. Finally, choose an arbitrary $\beta_{o}>0$, and define

$$
K_{\varepsilon}=\frac{1}{\varepsilon} \cdot \sqrt{\frac{1+\beta_{o}+4 \varepsilon^{4} / f^{\prime}(\mu)^{2}}{2 e \cdot \beta_{o}}}, \quad \beta_{\varepsilon}=\left(1+\beta_{o}\right) \cdot \lambda_{\varepsilon}^{\max }, \quad \text { and } \quad \gamma_{\varepsilon}=\gamma_{o} \cdot \lambda_{\varepsilon}^{\max },
$$

where $\lambda_{\varepsilon}^{\max }$ was defined in ([6). Then the following estimates hold:

$$
\begin{aligned}
& \left\|S_{\varepsilon}(t) v\right\|_{*} \leq K_{\varepsilon} \cdot t^{-1 / 2} \cdot e^{\beta_{\varepsilon} \cdot t} \cdot\|v\|_{L^{2}(\Omega)} \quad \text { for } \quad t>0, \quad v \in X, \\
& \left\|S_{\varepsilon}(t) v\right\|_{*} \leq e^{\lambda_{\varepsilon}^{\max } \cdot t} \cdot\|v\|_{*} \quad \text { for } \quad t \geq 0, \quad v \in X^{1 / 2}, \\
& \left\|S_{\varepsilon}(t) v^{+}\right\|_{*} \geq e^{\gamma_{\varepsilon} \cdot t} \cdot\left\|v^{+}\right\|_{*} \quad \text { for } \quad t \geq 0, \quad v^{+} \in X_{\varepsilon}^{+} \text {, } \\
& \left\|S_{\varepsilon}(t) v^{-}\right\|_{*} \leq e^{\gamma_{\varepsilon} \cdot t} \cdot\left\|v^{-}\right\|_{*} \quad \text { for } \quad t \geq 0, \quad v^{-} \in X_{\varepsilon}^{-} \text {. }
\end{aligned}
$$

The proof of the first two estimates can be found in Lemma 3.1 of [34, the remaining two estimates follow similarly. See also Lemma 3.4 in [29].

2.3. The Laplacian on rectangular domains. We collect a few definitions and results concerning the spectrum and the eigenfunctions of the negative Laplacian, which are easy consequences of (A1) and which will be needed later on. As in the Introduction, let $0<\kappa_{1} \leq \kappa_{2} \leq \ldots \rightarrow \infty$ denote the eigenvalues of the negative Laplacian $-\Delta: X \rightarrow X$ subject to homogeneous Neumann boundary conditions, and denote the corresponding complete set of $L^{2}(\Omega)$-orthonormalized eigenfunctions by $\psi_{1}, \psi_{2}, \ldots$. Notice that since we consider $-\Delta$ on the space $X$ of $L^{2}(\Omega)$-functions with total mass 0 defined in (3), the first eigenvalue $\kappa_{1}$ is strictly positive.

For the cubes $\Omega=(0,1)^{n}$ these eigenfunctions are given by all possible products of the form $\tilde{\psi}_{k_{1}}\left(x_{1}\right) \cdot \ldots \cdot \tilde{\psi}_{k_{n}}\left(x_{n}\right)$, where $\left(k_{1}, \ldots, k_{n}\right) \in \mathbb{N}_{0}^{n} \backslash\{(0, \ldots, 0)\}$,

$$
\tilde{\psi}_{0}(\tau)=1, \quad \text { and } \quad \tilde{\psi}_{k}(\tau)=\sqrt{2} \cdot \cos (k \pi \tau) \quad \text { for } \quad k \in \mathbb{N}, \tau \in(0,1) .
$$

The eigenvalue corresponding to $\tilde{\psi}_{k_{1}}\left(x_{1}\right) \cdot \ldots \cdot \tilde{\psi}_{k_{n}}\left(x_{n}\right)$ is given by $\left(k_{1}^{2}+\ldots+k_{n}^{2}\right) \cdot \pi^{2}$, and ordering these eigenvalues gives the sequence $\kappa_{k}$ from above. The case of arbitrary rectangular domains can be treated similarly.

In either case, the specific form of the eigenfunctions allows us to easily deduce the following properties. There exists a positive constant $C$ such that for all $k \in \mathbb{N}$ 
and arbitrary $i, j=1, \ldots, n$ we have

$$
\left\|\psi_{k}\right\|_{L^{\infty}(\Omega)} \leq C, \quad\left\|\frac{\partial \psi_{k}}{\partial x_{i}}\right\|_{L^{\infty}(\Omega)} \leq C \cdot \sqrt{\kappa_{k}}, \quad\left\|\frac{\partial^{2} \psi_{k}}{\partial x_{i} \partial x_{j}}\right\|_{L^{\infty}(\Omega)} \leq C \cdot \kappa_{k} .
$$

As for the asymptotic growth of the eigenvalues $\kappa_{k}$ one can show that

$$
\kappa_{k} \sim k^{2 / n} \quad \text { as } \quad k \rightarrow \infty .
$$

While the $\psi_{k}$ are orthonormalized with respect to the $L^{2}(\Omega)$-norm, our main phase space is given by the Hilbert space $X^{1 / 2}$ with norm $\|\cdot\|_{*}$. If we define

$$
\varphi_{k}=\frac{1}{\sqrt{1+\kappa_{k}^{2}}} \cdot \psi_{k} \quad \text { for } \quad k \in \mathbb{N}
$$

then the set $\varphi_{k}, k \in \mathbb{N}$, is a complete orthonormal set in this latter Hilbert space.

2.4. The dominating subspace. To close this section, we add a few remarks about the dominating subspace $X_{\varepsilon}^{+}$defined in (8). This definition, together with (5) and (6), implies that an eigenfunction $\psi_{k}$ is contained in the dominating subspace if and only if

(20) $\underline{\kappa}_{\varepsilon}=\frac{f^{\prime}(\mu)}{2 \varepsilon^{2}} \cdot\left(1-\sqrt{1-\gamma_{o}}\right) \leq \kappa_{k} \leq \bar{\kappa}_{\varepsilon}=\frac{f^{\prime}(\mu)}{2 \varepsilon^{2}} \cdot\left(1+\sqrt{1-\gamma_{o}}\right)$.

In other words, there are constants $N_{1, \varepsilon}, N_{2, \varepsilon} \in \mathbb{N}$ with $N_{1, \varepsilon} \leq N_{2, \varepsilon}$ such that

$$
X_{\varepsilon}^{+}=\operatorname{span}\left\{\psi_{k}: N_{1, \varepsilon} \leq k \leq N_{2, \varepsilon}\right\}=\operatorname{span}\left\{\varphi_{k}: N_{1, \varepsilon} \leq k \leq N_{2, \varepsilon}\right\} .
$$

Furthermore, due to (18) and (20) we have that

$$
\operatorname{dim} X_{\varepsilon}^{+} \sim \varepsilon^{-n} \quad \text { for } \quad \varepsilon \rightarrow 0, \quad \text { where } n=\operatorname{dim} \Omega .
$$

The fact that the dimension of the dominating subspace $X_{\varepsilon}^{+}$increases as $\varepsilon \rightarrow 0$ is one of the crucial factors in obtaining an accurate description of the patterns observed during spinodal decomposition. See Maier-Paape, Wanner [28]. On the other hand, it is also responsible for the dimension-dependent worst-case behavior in the results of 34, as we will demonstrate in Section 4.1.

\section{MAXIMUM NORMS OF RANDOM SUMS}

In this section we establish technical results on random sums of $C(\bar{\Omega})$-functions, which will be needed in Section 4.1. Throughout this section, $\Omega \subset \mathbb{R}^{n}$ denotes a bounded domain. The results are based on previous work by Aurich et al. 2 and Kahane [26]. In Section 3.1 we extend their studies by obtaining uniform bounds on certain time-dependent random sums. Section 3.2 contains auxiliary results which give lower bounds on the nodal domain sizes of functions in the dominating subspace $X_{\varepsilon}^{+}$defined in (8).

3.1. Random time-dependent sums. Throughout this section, we consider the following situation.

Definition 3.1. Let $\Omega \subset \mathbb{R}^{n}$ be an open and bounded domain, and let $\left\{w_{1}, \ldots, w_{N}\right\}$ be a finite set of $C(\bar{\Omega})$-functions. We assume that the constant $M_{1}$ is such that

$$
\left\|w_{k}\right\|_{L^{\infty}(\Omega)} \leq M_{1} \quad \text { for all } \quad k=1, \ldots, N .
$$


In addition, let $c_{1}, \ldots, c_{N}$ be arbitrary real numbers, and let $\mu_{1}, \ldots, \mu_{N}$ be nonnegative real numbers. For some $T>0$ we consider functions of the form

$$
w(t, x)=\sum_{k=1}^{N} e^{-\mu_{k} \cdot t} \cdot c_{k} \cdot w_{k}(x) \quad \text { for all } \quad t \in[0, T] \quad \text { and } \quad x \in \Omega .
$$

Finally, let $\Omega_{T}=[0, T] \times \Omega$ and define

$$
\mathcal{M}(w)=\left\{(t, x) \in[0, T] \times \Omega:|w(t, x)| \geq\|w\|_{L^{\infty}\left(\Omega_{T}\right)} / 2\right\} .
$$

The main result of this section provides a sharp upper bound on the $L^{\infty}\left(\Omega_{T}\right)$ norm of time-dependent functions $w$ as defined in (23). For this, we need to make sure that the set $\mathcal{M}(w)$ defined in (24) cannot be too small. This is addressed in the following assumption. For our application to the Cahn-Hilliard equation we verify its validity in Section 3.2 .

Assumption 3.2. In the situation of Definition 3.1 we assume that there exists a constant $\varrho>0$ which depends only on the constants $\mu_{k}$ and the functions $w_{k}$, such that for every choice of $c_{1}, \ldots, c_{N} \in \mathbb{R}$ the function $w$ defined in (23) satisfies

$$
\operatorname{vol}_{n+1}(\mathcal{M}(w)) \geq \varrho \cdot \operatorname{vol}_{n+1}\left(\Omega_{T}\right),
$$

where vol $_{\ell}$ denotes $\ell$-dimensional Lebesgue measure.

The following lemma is an extension of similar results due to Aurich et al. [2] Theorem 1] and Kahane [26, Section 6.2, Theorem 1]. Unlike their situations, we obtain uniform $L^{\infty}\left(\Omega_{T}\right)$-bounds for random one-parameter families as in (23).

Lemma 3.3. Assume the situation of Definition 3.1 and let $\varrho$ be as in Assumption 3.2 Let $a_{1}, \ldots, a_{N}$ denote independent random variables over a probability space $(F, \mathcal{F}, \mathbb{P})$ which are all normally distributed with mean 0 and variance 1 . We consider random functions of the form

$$
w(t, x, \omega)=\sum_{k=1}^{N} e^{-\mu_{k} \cdot t} \cdot a_{k}(\omega) \cdot w_{k}(x),
$$

where $(t, x) \in \Omega_{T}=[0, T] \times \Omega$, and $\omega \in F$. Then for any $\varrho^{*}>\varrho / 2$ we have

$$
\mathbb{P}\left(\|w\|_{L^{\infty}\left(\Omega_{T}\right)} \geq M_{1} \cdot \sqrt{8 N \cdot \ln \left(2 \varrho^{*} / \varrho\right)}\right) \leq \frac{1}{\varrho^{*}} .
$$

Proof. Since each random variable $a_{k}$ is normally distributed with mean 0 and variance 1 we obtain for every $\tau \in \mathbb{R}$ and $k=1, \ldots, N$ that the expected value $\mathbb{E}$ of the random variable $e^{\tau a_{k}}$ satisfies

$$
\mathbb{E}\left(e^{\tau \cdot a_{k}}\right)=\int_{F} e^{\tau \cdot a_{k}(\omega)} d \mathbb{P}=e^{\tau^{2} / 2}
$$

see for example Bauer [5]. Now let $\alpha \in \mathbb{R}$ be arbitrary and let $k \in\{1, \ldots, N\}$. Then for all $t \in[0, T]$ and $x \in \Omega$ we have due to $\mu_{k} \geq 0$ that

$$
\mathbb{E}\left(e^{\alpha \cdot e^{-\mu_{k} \cdot t} \cdot a_{k} \cdot w_{k}(x)}\right)=e^{\alpha^{2} \cdot e^{-2 \mu_{k} \cdot t} \cdot w_{k}(x)^{2} / 2} \leq e^{\alpha^{2} \cdot\left\|w_{k}\right\|_{L}^{2 \infty(\Omega)} / 2},
$$

and the independence of the $a_{k}$ 's furnishes

$$
\mathbb{E}\left(e^{\alpha \cdot w(t, x, \cdot)}\right)=\prod_{k=1}^{N} \mathbb{E}\left(e^{\alpha \cdot e^{-\mu_{k} \cdot t} \cdot a_{k} \cdot w_{k}(x)}\right) \leq e^{\alpha^{2} \cdot M_{1}^{2} \cdot N / 2},
$$


where $M_{1}$ was defined in Definition 3.1. From now on, we assume $\alpha \geq 0$. Then for any $t \in[0, T]$ the estimate (25) implies

$$
\begin{aligned}
& \varrho \cdot \operatorname{vol}_{n+1}\left(\Omega_{T}\right) \cdot \mathbb{E}\left(e^{\alpha \cdot\|w\|_{L} \infty_{\left(\Omega_{T}\right)} / 2}\right) \leq \mathbb{E}\left(\int_{\mathcal{M}(w)} e^{\alpha \cdot|w(t, x, \cdot)|} d(t, x)\right) \\
\leq & \mathbb{E}\left(\int_{\mathcal{M}(w)}\left(e^{\alpha \cdot w(t, x, \cdot)}+e^{-\alpha \cdot w(t, x, \cdot)}\right) d(t, x)\right) \\
\leq & \int_{[0, T] \times \Omega} \mathbb{E}\left(e^{\alpha \cdot w(t, x, \cdot)}+e^{-\alpha \cdot w(t, x, \cdot)}\right) d(t, x) \leq 2 \cdot T \cdot \operatorname{vol}_{n}(\Omega) \cdot e^{\alpha^{2} \cdot M_{1}^{2} \cdot N / 2},
\end{aligned}
$$

which furnishes the inequality

$$
\mathbb{E}\left(e^{\alpha \cdot\|w\|_{L}\left(\Omega_{T}\right) / 2}\right) \leq \frac{2}{\varrho} \cdot e^{\alpha^{2} \cdot M_{1}^{2} \cdot N / 2} .
$$

Notice that in the above estimates, both the set $\mathcal{M}(w)$ and the norm $\|w\|_{L^{\infty}\left(\Omega_{T}\right)}$ depend on $\omega$. For any $\varrho^{*}>\varrho / 2$ the last inequality can be rewritten as

$$
\mathbb{E}\left(\exp \left(\frac{\alpha}{2} \cdot\left(\|w\|_{L^{\infty}\left(\Omega_{T}\right)}-\alpha \cdot M_{1}^{2} \cdot N-\frac{2}{\alpha} \cdot \ln \left(2 \varrho^{*} / \varrho\right)\right)\right)\right) \leq \frac{1}{\varrho^{*}} .
$$

Since for any random variable $\xi$ and any $\alpha \geq 0$ one has

$$
\mathbb{P}(\xi \geq 0) \leq \int_{\{\xi \geq 0\}} e^{\alpha \cdot \xi / 2} d \mathbb{P} \leq \mathbb{E}\left(e^{\alpha \cdot \xi / 2}\right)
$$

we obtain

$$
\mathbb{P}\left(\|w\|_{L^{\infty}\left(\Omega_{T}\right)} \geq \alpha \cdot M_{1}^{2} \cdot N+\frac{2}{\alpha} \cdot \ln \left(2 \varrho^{*} / \varrho\right)\right) \leq \frac{1}{\varrho^{*}} .
$$

Choosing $\alpha=\left(2 \cdot \ln \left(2 \varrho^{*} / \varrho\right) \cdot M_{1}^{-2} \cdot N^{-1}\right)^{1 / 2}$ this finally yields (27), and the proof of the lemma is complete.

The above lemma could easily be generalized. Similar to the results in Aurich et al. 2] and Kahane [26, it suffices to assume that the random variables $a_{i}$ are independent and subnormally distributed. However, for our purposes the above formulation is more adequate.

The following result combines Lemma 3.3 with the weak law of large numbers to relate the maximum norm of the function $w$ to the standard Euclidean norm of the coefficient vector $\left(a_{1}, \ldots, a_{N}\right)$. In our application to the Cahn-Hilliard equation, this Euclidean norm corresponds basically to the $H^{2}(\Omega)$-norm of $w(0, \cdot)$.

Proposition 3.4. Assume the situation of Definition 3.1, let $\varrho$ be as in Assumption 3.2, let $\varrho^{*}>\varrho / 2$, and let $a_{1}, \ldots, a_{N}$ be independent random variables over a common probability space $(F, \mathcal{F}, \mathbb{P})$ which are all normally distributed with mean 0 and variance 1 . We consider again random functions of the form (26). Then there exists a set $F_{0} \in \mathcal{F}$ satisfying

$$
\mathbb{P}\left(F_{0}\right) \geq 1-\frac{1}{\varrho^{*}}-\frac{8}{N}
$$

such that for every $\omega \in F_{0}$ and all $t \in[0, T]$ we have

$$
\|w(t, \cdot, \omega)\|_{L^{\infty}(\Omega)} \leq 4 M_{1} \cdot \sqrt{\ln \left(2 \varrho^{*} / \varrho\right)} \cdot|a(\omega)|,
$$

where $a(\omega)=\left(a_{1}(\omega), \ldots, a_{N}(\omega)\right) \in \mathbb{R}^{N}$. 
Proof. According to the distribution assumptions on the independent random variables $a_{n}$ we have $\mathbb{E}\left(|a|^{2}\right)=\mathbb{E}\left(a_{1}^{2}+\ldots+a_{N}^{2}\right)=N$. Furthermore, one can easily compute the variance of $|a|^{2}$ as $\mathbb{V}\left(|a|^{2}\right)=2 N$. See for example Bauer [5, §4]. The weak law of large numbers, in the form of Chebyshev's inequality, then furnishes

$$
\mathbb{P}\left(\left.|| a\right|^{2}-N \mid \geq \frac{N}{2}\right) \leq \frac{4}{N^{2}} \cdot \mathbb{V}\left(|a|^{2}\right)=\frac{8}{N},
$$

and therefore we have

$$
\mathbb{P}\left(|a| \leq \sqrt{\frac{N}{2}}\right) \leq \frac{8}{N} .
$$

Now let $F_{0} \in \mathcal{F}$ consist of all those $\omega \in F$ for which both $|a(\omega)|>\sqrt{N / 2}$ and $\sup _{t \in[0, T]}\|w(t, \cdot, \omega)\|_{L^{\infty}(\Omega)}<M_{1} \cdot \sqrt{8 N \cdot \ln \left(2 \varrho^{*} / \varrho\right)}$ are satisfied. Due to (29) and Lemma 3.3 we have $\mathbb{P}\left(F_{0}\right) \geq 1-1 / \varrho^{*}-8 / N$, which completes the proof of the proposition.

We are finally in a position to prove our main result on the maximum norms of random sums. The formulation below replaces the random, normally distributed coefficients by vectors chosen from a sphere in $\mathbb{R}^{N}$. It shows that for most of these vectors, with respect to the uniform measure on the sphere, sharp estimates on the maximum norm can be obtained. This formulation is exactly what is needed for our application to spinodal decomposition in Section 4

Theorem 3.5. Assume the situation of Definition 3.1 let $\varrho$ be as in Assumption 3.2 and for $R>0$ let $S_{R}=\left\{a \in \mathbb{R}^{N}:|a|=R\right\}$ denote the sphere of radius $R$. Finally, let $m_{R}$ denote the (unique) uniform Haar probability measure on $S_{R}$ and let $\varrho^{*}>\varrho / 2$. Then there exists a measurable set $S^{*} \subset S_{R}$ with $m_{R}\left(S^{*}\right) \geq 1-1 / \varrho^{*}-8 / N$ such that the following is true. If $a=\left(a_{1}, \ldots, a_{N}\right) \in S^{*}$ and if $w$ is defined as

$$
w(t, x)=\sum_{k=1}^{N} e^{-\mu_{k} \cdot t} \cdot a_{k} \cdot w_{k}(x),
$$

then we have

$$
\|w(t, \cdot)\|_{L^{\infty}(\Omega)} \leq 4 M_{1} \cdot \sqrt{\ln \left(2 \varrho^{*} / \varrho\right)} \cdot|a| \quad \text { for all } \quad t \in[0, T] .
$$

Proof. The result follows immediately from Proposition 3.4 One just has to recall two facts. First of all, if (28) holds for some coefficient vector $\left(a_{1}, \ldots, a_{N}\right)$, then it also holds for any multiple of that coefficient vector. Secondly, if $a_{1}, \ldots, a_{N}$ are independent random variables over a common probability space $(F, \mathcal{F}, \mathbb{P})$ such that all of them are normally distributed with mean 0 and variance 1 , then the mapping

$$
F \ni \omega \mapsto \frac{R}{|a(\omega)|} \cdot a(\omega) \in S_{R}
$$

maps the measure $\mathbb{P}$ to the Haar measure $m_{R}$ on $S_{R}$. This last statement follows easily from the properties of Gaussian measures as described in Bauer [5, §30], in combination with the uniqueness of the Haar measure $m_{R}$ on $S_{R}$ established in Cohn [12 Chapter 9]. See also Muirhead [30, Section 1.5]. Thus, the set $S^{*}$ can be chosen as the image of the set $F_{0}$ from Proposition 3.4 under this mapping. 
3.2. A lower bound on the nodal domain size. In this section we establish the validity of Assumption 3.2 for a large class of functions of the form (23). We begin with an abstract result, which is then used later to cover the special cases necessary for our applications to the Cahn-Hilliard equation in Section 4. The abstract result provides a bound on the constant $\varrho$ in (25) depending on $L^{\infty}(\Omega)$-bounds for the functions $w_{k}$ and their derivatives. More precisely, we have to assume the following.

Assumption 3.6. Let $\Omega$ denote a bounded domain in $\mathbb{R}^{n}$, where $n \in\{1,2,3\}$. Moreover, let $\left\{w_{1}, \ldots, w_{N}\right\}$ be a finite set of $L^{2}(\Omega)$-orthonormal functions which are all contained in $C^{1}(\bar{\Omega})$, and let $M_{1}$ and $M_{2}$ be constants satisfying

$$
\left\|w_{k}\right\|_{L^{\infty}(\Omega)} \leq M_{1} \quad \text { and } \quad\left\|\nabla w_{k}\right\|_{L^{\infty}(\Omega)} \leq M_{2} \quad \text { for } \quad k=1, \ldots, N .
$$

In order to simplify notation we write $\left\|\nabla w_{k}\right\|_{L^{\infty}(\Omega)}$ instead of $\left\|\left|\nabla w_{k}\right|\right\|_{L^{\infty}(\Omega)}$. Finally, assume that the domain $\Omega$ satisfies the cone condition [1, 4.3]: There exists a finite cone $\mathcal{C}$ such that each point $x \in \Omega$ is the vertex of a cone $\mathcal{C}_{x}$ contained in $\Omega$ and congruent to $\mathcal{C}$. We denote the height of $\mathcal{C}$ by $r_{\mathcal{C}}$. (This height corresponds to the radius of the ball $B_{1}$ in Adams [1, 4.1].)

The next result establishes bounds on the constant $\varrho$ in Assumption 3.2, Notice that for the result, the $L^{2}(\Omega)$-orthonormality of the functions $w_{k}$ assumed above is essential.

Lemma 3.7. Suppose that Assumption 3.6 is satisfied, and let $\mu_{1}, \ldots, \mu_{N}$ be nonnegative numbers satisfying $0 \leq \mu_{k} \leq M_{3}$ for all $k=1, \ldots, N$, for some constant $M_{3}$. Furthermore, let $T>0$ be fixed, and assume that the constant $r$ defined as

$$
r=N^{-1} \cdot\left(8 \cdot \operatorname{vol}_{n}(\Omega) \cdot\left(M_{1}^{2} M_{3}^{2}+M_{2}^{2}\right)\right)^{-1 / 2}
$$

satisfies $r \leq \min \left\{r_{\mathcal{C}}, T\right\}$. Let $w$ be any function of the form (23). Then we have

$$
\operatorname{vol}_{n+1}(\mathcal{M}(w)) \geq r^{n+1} \cdot \frac{\operatorname{vol}_{n}(\mathcal{C})}{r_{\mathcal{C}}^{n}}
$$

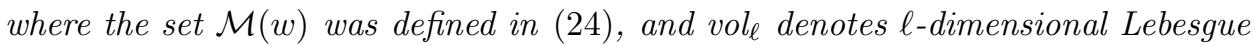
measure.

Proof. The orthonormality of the $w_{k}$ 's furnishes $\left(w(0, \cdot), w_{k}\right)_{L^{2}(\Omega)}=c_{k}$, and therefore we obtain for every $k=1, \ldots, N$ the estimate

$$
\begin{aligned}
\left|c_{k}\right| & =\left|\left(w(0, \cdot), w_{k}\right)_{L^{2}(\Omega)}\right| \leq\|w(0, \cdot)\|_{L^{\infty}(\Omega)} \cdot \int_{\Omega}\left|w_{k}\right| d x \\
& \leq\|w\|_{L^{\infty}\left(\Omega_{T}\right)} \cdot \sqrt{\operatorname{vol}_{n}(\Omega)} \cdot\left\|w_{k}\right\|_{L^{2}(\Omega)}=\sqrt{\operatorname{vol}_{n}(\Omega)} \cdot\|w\|_{L^{\infty}\left(\Omega_{T}\right)} .
\end{aligned}
$$

For $(t, x) \in[0, T] \times \Omega$ this estimate implies

$$
\left|\frac{\partial w}{\partial t}(t, x)\right| \leq \sum_{k=1}^{N} \mu_{k} \cdot e^{-\mu_{k} \cdot t} \cdot\left|c_{k}\right| \cdot\left|w_{k}(x)\right| \leq N \cdot M_{1} M_{3} \cdot \sqrt{\operatorname{vol}_{n}(\Omega)} \cdot\|w\|_{L^{\infty}\left(\Omega_{T}\right)},
$$

as well as

$$
|\nabla w(t, x)| \leq \sum_{k=1}^{N} e^{-\mu_{k} \cdot t} \cdot\left|c_{k}\right| \cdot\left|\nabla w_{k}(x)\right| \leq N \cdot M_{2} \cdot \sqrt{\operatorname{vol}_{n}(\Omega)} \cdot\|w\|_{L^{\infty}\left(\Omega_{T}\right)},
$$


where the gradient $\nabla=\nabla_{x}$ is taken with respect to $x$ only. Together these estimates yield

$$
\left|\nabla_{(t, x)} w(t, x)\right| \leq N \cdot \sqrt{\operatorname{vol}_{n}(\Omega) \cdot\left(M_{1}^{2} M_{3}^{2}+M_{2}^{2}\right)} \cdot\|w\|_{L^{\infty}\left(\Omega_{T}\right)} .
$$

Now let $\left(t_{*}, x_{*}\right) \in[0, T] \times \bar{\Omega}$ be such that $\left|w\left(t_{*}, x_{*}\right)\right|=\|w\|_{L^{\infty}\left(\Omega_{T}\right)}$. Fix an interval $I_{*} \subset[0, T]$ of length $r$ which contains $t_{*}$, and let $\mathcal{C}_{*} \subset \Omega$ denote a cone with vertex $x_{*}$ which is congruent to $\left(r / r_{\mathcal{C}}\right) \cdot \mathcal{C}$. Then for all $(t, x) \in I_{*} \times \mathcal{C}_{*}$ we have

$$
\begin{aligned}
\left|w(t, x)-w\left(t_{*}, x_{*}\right)\right| & \leq \sup _{(t, x) \in \Omega_{T}}\left|\nabla_{(t, x)} w(t, x)\right| \cdot \sqrt{\left|t-t_{*}\right|^{2}+\left|x-x_{*}\right|^{2}} \\
& \leq N \cdot \sqrt{\operatorname{vol}_{n}(\Omega) \cdot\left(M_{1}^{2} M_{3}^{2}+M_{2}^{2}\right)} \cdot\|w\|_{L^{\infty}\left(\Omega_{T}\right)} \cdot \sqrt{r^{2}+r^{2}} \\
& =\frac{1}{2} \cdot\|w\|_{L^{\infty}\left(\Omega_{T}\right)} .
\end{aligned}
$$

This implies $I_{*} \times \mathcal{C}_{*} \subset \mathcal{M}(w)$, and noting that $\operatorname{vol}_{n+1}\left(I_{*} \times \mathcal{C}_{*}\right)=r \cdot\left(r / r_{\mathcal{C}}\right)^{n} \cdot \operatorname{vol}_{n}(\mathcal{C})$ completes the proof of the lemma.

Remark 3.8. The statement of this lemma could easily be improved, even though this is not necessary for our applications. If the assumption $r \leq \min \left\{r_{\mathcal{C}}, T\right\}$ is not satisfied, let $r_{*}=\min \left\{r, r_{\mathcal{C}}\right\}$ and $T_{*}=\min \{r, T\}$. Then (31) can be replaced by the estimate $\operatorname{vol}_{n+1}(\mathcal{M}(w)) \geq T_{*} \cdot r_{*}^{n} \cdot r_{\mathcal{C}}^{-n} \cdot \operatorname{vol}_{n}(\mathcal{C})$.

The following corollary establishes the asymptotic behavior of the constant $\varrho$ in (25) for our applications to transient pattern formation in the Cahn-Hilliard equation. For this we need to consider one-parameter families which are contained in the dominating subspace $X_{\varepsilon}^{+}$defined in (8). Recall that in Section 2.4 we showed that there are constants $N_{1, \varepsilon}, N_{2, \varepsilon} \in \mathbb{N}$ with $N_{1, \varepsilon} \leq N_{2, \varepsilon}$ such that (21) holds. Using this notation, we obtain the following result.

Corollary 3.9. Assume that (A1) is satisfied, let $N_{1, \varepsilon}, N_{2, \varepsilon}$ be as in (21), and let $M_{4}$ and $T$ denote positive constants. Then there exists an $\varepsilon_{o}>0$ and a constant $M_{5}>0$ such that for all $0<\varepsilon \leq \varepsilon_{o}$ the following holds. Let $\mu_{N_{1, \varepsilon}}, \ldots, \mu_{N_{2, \varepsilon}}$ be arbitrary constants satisfying

$$
0 \leq \mu_{k} \leq \frac{M_{4}}{\varepsilon^{2}} \quad \text { for all } \quad N_{1, \varepsilon} \leq k \leq N_{2, \varepsilon},
$$

let $c_{N_{1, \varepsilon}}, \ldots, c_{N_{2, \varepsilon}} \in \mathbb{R}$ be arbitrary, and consider the one-parameter family

$$
w(t, \cdot)=\sum_{k=N_{1, \varepsilon}}^{N_{2, \varepsilon}} e^{-\mu_{k} \cdot t} \cdot c_{k} \cdot \psi_{k}(\cdot) \in X_{\varepsilon}^{+} \quad \text { for } \quad t \in[0, T] .
$$

Then we have

(34) $\operatorname{vol}_{n+1}\left(\mathcal{M}\left(\partial w / \partial x_{i}\right)\right) \geq M_{5} \cdot \varepsilon^{(n+1)(n+2)} \cdot \operatorname{vol}_{n+1}\left(\Omega_{T}\right), \quad i=1, \ldots, n$,

where $\Omega_{T}=[0, T] \times \Omega$, the set $\mathcal{M}(w)$ was defined in (24), and vol denotes $\ell$ dimensional Lebesgue measure.

Proof. The first estimate (33) follows easily from the previous result if we note that according to Section 2.3. $M_{1}$ can be chosen constant and $M_{2}$ proportional to $\varepsilon^{-1}$. In addition, (32) implies that $M_{3}$ is proportional to $\varepsilon^{-2}$. Since $N$ equals the dimension 
of the dominating subspace $X_{\varepsilon}^{+}$, it is proportional to $\varepsilon^{-n}$ due to (22). Lemma 3.7 now implies (33).

As for the estimates for the partial derivatives of $w$ we note that the set of those functions $\partial \psi_{k} / \partial x_{i}$, which are not identically zero, still forms an orthogonal set in $L^{2}(\Omega)$; the only difference is that one of the cosine factors is replaced by a scalar multiple of the sine. After normalizing these functions and adjusting the coefficients $c_{k}$, we can choose the constants necessary for Lemma 3.7 as above, and (34) follows.

The above result is the main reason for considering only rectangular domains for the case of the Cahn-Hilliard equation. While the validity of (33) can be established easily for more general domains, the estimates (34) for the partial derivatives of the eigenfunctions cannot be obtained by the above method - the partial derivatives are in general no longer an orthogonal set in $L^{2}(\Omega)$. Once this problem can be resolved, our results could be generalized immediately.

\section{Application to transient PATtern FORMATion}

In this main section of the paper, we apply the abstract results on maximum norms of random sums to transient pattern formation in the nonlinear Cahn-Hilliard equation. We assume the setting described in Section 2.

4.1. Sharp bounds on the nonlinearity. This section is devoted to obtaining sharp estimates on the nonlinearity of the Cahn-Hilliard equation. Our approach is based on the one by Sander and Wanner [34], and therefore we briefly describe their results. Assuming (A3) from Section 2.1, the main result of [34] establishes bounds on the nonlinearity $F$ of the Cahn-Hilliard equation in certain cones. Consider the dominating subspace $X_{\varepsilon}^{+}$defined in (8), and let $X_{\varepsilon}^{-}$denote its orthogonal complement in $X^{1 / 2}$. For any $\delta>0$, define the cone

$$
\mathcal{K}_{\varepsilon, \delta}=\left\{u \in X^{1 / 2}:\left\|u^{-}\right\|_{*} \leq \delta \cdot\left\|u^{+}\right\|_{*}, \quad u=u^{+}+u^{-} \in X_{\varepsilon}^{+} \oplus X_{\varepsilon}^{-}\right\} .
$$

The following result was proved in 34 .

Lemma 4.1 (Lemma 3.3 in [34]). Assume that (A1) and (A3) are satisfied, and let $F$ be defined as in (15). Let $\delta_{o}>0$ be arbitrary, and set

$$
\delta_{\varepsilon}=\delta_{o} \cdot \varepsilon^{2-n / 2} .
$$

Then there exist $\varepsilon$-independent constants $M_{1}, M_{2}>0$ such that for every $\varepsilon \in(0,1)$ and every function $u \in \mathcal{K}_{\varepsilon, \delta_{\varepsilon}}$ with $\|u\|_{*} \leq M_{1} \cdot \varepsilon^{-2+n / 2}$ we have

$$
\|F(u)\|_{L^{2}(\Omega)} \leq M_{2} \cdot \varepsilon^{(2-n / 2) \cdot \sigma} \cdot\|u\|_{*}^{\sigma+1} .
$$

This result was the crucial ingredient for improving the spinodal decomposition results by Maier-Paape and Wanner [28, 29]. It states that close to the dominating subspace $X_{\varepsilon}^{+}$, the nonlinearity remains extremely small, even at large distances from the homogeneous equilibrium. Central for establishing Lemma 4.1 is an estimate which relates the $L^{\infty}(\Omega)$-norm of functions in $X_{\varepsilon}^{+}$to their $X^{1 / 2}$-norm. More precisely, it is shown in 34 that

$$
\|v\|_{L^{\infty}(\Omega)} \leq C \cdot \varepsilon^{2-n / 2} \cdot\|v\|_{*} \quad \text { for all } \quad v \in X_{\varepsilon}^{+} .
$$

Any improvement of this estimate would translate directly into an improvement of Lemma 4.1, and therefore also of the order of the maximal radius in Theorem 1.1. 
As we already indicated in the Introduction, such an improvement is impossible. Consider for example the case $\Omega=(0,1)^{n}$, which was described in Section [2.3. In this situation the eigenfunctions of the negative Laplacian are given by products of cosines. Furthermore, an eigenfunction $\psi_{k}$ is contained in the dominating subspace $X_{\varepsilon}^{+}$of (8) if and only if (20) holds. If we now let $N_{\varepsilon}=\operatorname{dim} X_{\varepsilon}^{+}$and define a function $w_{\varepsilon} \in X_{\varepsilon}^{+}$by

$$
w_{\varepsilon}=\frac{1}{N_{\varepsilon}} \cdot \sum_{\underline{\kappa}_{\varepsilon} \leq \kappa_{k} \leq \bar{\kappa}_{\varepsilon}} \psi_{k}
$$

then (19) implies $\left(1+\underline{\kappa}_{\varepsilon}^{2}\right) / N_{\varepsilon} \leq\left\|w_{\varepsilon}\right\|_{*}^{2} \leq\left(1+\bar{\kappa}_{\varepsilon}^{2}\right) / N_{\varepsilon}$. Since for every $k \in \mathbb{N}$ the eigenfunction $\psi_{k}$ is the product of $n$ functions of the form (17), its function value at the origin equals its $L^{\infty}(\Omega)$-norm, which is given by $2^{n_{k} / 2}$ for some $1 \leq n_{k} \leq n$. The above definition of $w_{\varepsilon}$ then furnishes $\sqrt{2} \leq\left\|w_{\varepsilon}\right\|_{L^{\infty}(\Omega)} \leq 2 \sqrt{2}$, and together with (20) and (22) readily implies the existence of two $\varepsilon$-independent constants $c_{1}$ and $c_{2}$ such that

$$
c_{1} \cdot \varepsilon^{2-n / 2} \leq \frac{\left\|w_{\varepsilon}\right\|_{L^{\infty}(\Omega)}}{\left\|w_{\varepsilon}\right\|_{*}} \leq c_{2} \cdot \varepsilon^{2-n / 2}
$$

This estimate proves the optimality of (36) for $\Omega=(0,1)^{n}$, and it is not hard to show that for the functions defined in (37) the nonlinearity estimate in Lemma 4.1 cannot be improved either. Finally, recall that the numerical simulations presented in Figure 3 already indicated the optimality of the main result of [34.

In order to explain why for most randomly selected initial conditions linear behavior determines the dynamics for much further, we therefore have to restrict our attention to a suitable subset of the cone $\mathcal{K}_{\varepsilon, \delta_{\varepsilon}}$ considered in Lemma4.1. The goal of such a study must be the explanation of the orders suggested by the simulations in Figure 2- and this is the subject of the current section.

As a first step towards obtaining an improved estimate on the nonlinearity $F$ we have to study the temporal evolution of the $L^{\infty}(\Omega)$-norm of certain solutions (and their gradients) of the linearized Cahn-Hilliard equation (2). It was mentioned in Section 2.2 that $A_{\varepsilon}$ generates an analytic semigroup $S_{\varepsilon}(t), t \geq 0$, on $X$. The unique solution of (2) originating at $v_{o}$ is given by the formula $v(t)=S_{\varepsilon}(t) v_{o}$, with explicit representation (16). We are particularly interested in solutions of (2) for which $v_{o}$ is contained in the dominating subspace $X_{\varepsilon}^{+}$. The next lemma is the key for obtaining our sharp nonlinearity estimates.

Lemma 4.2. Let $\Omega$ be as in (A1) and let $T>0$ be arbitrary. Then there exist $\varepsilon$-independent constants $C_{1}$ and $C_{2}$, and for every sufficiently small $\varepsilon>0$ there exists a subset $\mathcal{G}_{\varepsilon}^{+} \subset X_{\varepsilon}^{+}$such that the following hold:

(a) For any $v^{+} \in \mathcal{G}_{\varepsilon}^{+}$and arbitrary $r>0$ we have $r \cdot v^{+} \in \mathcal{G}_{\varepsilon}^{+}$, i.e., the set $\mathcal{G}_{\varepsilon}^{+}$ is the union of half-rays originating at the origin.

(b) Let $r>0$ be arbitrary, and let $m_{r}$ denote the uniform probability measure on the sphere $S_{\varepsilon, r}^{+}=\left\{v^{+} \in X_{\varepsilon}^{+}:\left\|v^{+}\right\|_{*}=r\right\}$. Then

$$
m_{r}\left(\mathcal{G}_{\varepsilon}^{+} \cap S_{\varepsilon, r}^{+}\right) \geq 1-C_{1} \cdot \varepsilon^{n} .
$$


(c) Let $v_{o}^{+} \in \mathcal{G}_{\varepsilon}^{+}$be arbitrary, and let $v^{+}(t)=S_{\varepsilon}(t) v_{o}^{+}$denote the solution of the linear equation (2) starting at $v_{o}^{+}$. Then for all $0 \leq t \leq T$ we have

$$
\begin{aligned}
\left\|S_{\varepsilon}(t) v_{o}^{+}\right\|_{L^{\infty}(\Omega)} & \leq C_{2} \cdot \varepsilon^{2} \cdot \sqrt{|\ln \varepsilon|} \cdot e^{\lambda_{\varepsilon}^{\max } \cdot t} \cdot\left\|v_{o}^{+}\right\|_{*}, \\
\left\|\nabla S_{\varepsilon}(t) v_{o}^{+}\right\|_{L^{\infty}(\Omega)} & \leq C_{2} \cdot \varepsilon \cdot \sqrt{|\ln \varepsilon|} \cdot e^{\lambda_{\varepsilon}^{\max } \cdot t} \cdot\left\|v_{o}^{+}\right\|_{*}, \\
\left\|\nabla S_{\varepsilon}(t) v_{o}^{+}\right\|_{L^{4}(\Omega)} & \leq C_{2} \cdot \varepsilon \cdot \sqrt[4]{|\ln \varepsilon|} \cdot e^{\lambda_{\varepsilon}^{\max } \cdot t} \cdot\left\|v_{o}^{+}\right\|_{*},
\end{aligned}
$$

where we write $\left\|\nabla S_{\varepsilon}(t) v_{o}^{+}\right\|_{L^{\infty}(\Omega)}$ instead of $\left\|\left|\nabla S_{\varepsilon}(t) v_{o}^{+}\right|\right\|_{L^{\infty}(\Omega)}$ to simplify the notation, and similarly for $\left\|\nabla S_{\varepsilon}(t) v_{o}^{+}\right\|_{L^{4}(\Omega)}$.

Proof. It suffices to construct a subset $\mathcal{G}_{\varepsilon, 1}^{+}$of the unit sphere $S_{\varepsilon, 1}^{+}$in $X_{\varepsilon}^{+}$such that (b) is satisfied with $r=1$ and such that (c) holds for all $v_{o}^{+} \in \mathcal{G}_{\varepsilon, 1}^{+}$. If we then define

$$
\mathcal{G}_{\varepsilon}^{+}=\bigcup_{r>0}\left(r \cdot \mathcal{G}_{\varepsilon, 1}^{+}\right) \subset X_{\varepsilon}^{+},
$$

(a) is automatically satisfied, and the validity of (b) for arbitrary $r>0$ follows easily. Furthermore, since we are considering the evolution of a linear semigroup, the estimates in (c) remain valid for arbitrary $v_{o}^{+} \in \mathcal{G}_{\varepsilon}^{+}$.

The set $\mathcal{G}_{\varepsilon, 1}^{+}$is constructed using the results on maximum norms of random sums derived in Section 3. We begin by establishing the first estimate (38). According to the definition of the dominating subspace $X_{\varepsilon}^{+}$and (5) there exist integers $N_{1, \varepsilon} \leq N_{2, \varepsilon}$ such that (21) holds. Now choose $\left\{w_{1}, \ldots, w_{N}\right\}$ in Definition 3.1 as $\left\{\varphi_{N_{1, \varepsilon}}, \ldots, \varphi_{N_{2, \varepsilon}}\right\}$, where $\varphi_{k}$ was defined in (19). Due to (22), we obtain that $N=N_{2, \varepsilon}-N_{1, \varepsilon}+1$ is bounded above by $C \cdot \varepsilon^{-n}$. Moreover, the constant $M_{1}$ in Definition 3.1 is bounded above by $C \cdot \varepsilon^{2}$ due to (19) and (20). For $c_{N_{1, \varepsilon}}, \ldots, c_{N_{2, \varepsilon}} \in \mathbb{R}$ we consider functions of the form

$$
w(t, \cdot)=e^{-\lambda_{\varepsilon}^{\max } \cdot t} \cdot S_{\varepsilon}(t) v_{o}^{+}=\sum_{k=N_{1, \varepsilon}}^{N_{2, \varepsilon}} e^{-\left(\lambda_{\varepsilon}^{\max }-\lambda_{k, \varepsilon}\right) \cdot t} \cdot c_{k} \cdot \varphi_{k},
$$

where $v_{o}^{+}=\sum_{k=N_{1, \varepsilon}}^{N_{2, \varepsilon}} c_{k} \varphi_{k} \in X_{\varepsilon}^{+}$. Then we have $\left\|v_{o}^{+}\right\|_{*}=\left|\left(c_{N_{1, \varepsilon}}, \ldots, c_{N_{2, \varepsilon}}\right)\right|$, and the factors $\lambda_{\varepsilon}^{\max }-\lambda_{k, \varepsilon}$ in the exponents of the exponential terms are bounded by $C \cdot \varepsilon^{-2}$.

Now we have gathered everything to employ the results of Section 3 Corollary 3.9 implies that $\varrho$ in Theorem 3.5 can be chosen as $C \cdot \varepsilon^{(n+1)(n+2)}$. If we now apply Theorem 3.5 with $R=1$ and $\varrho^{*}=\varepsilon^{-n}$, then (30) readily furnishes (38) on a set $\mathcal{N}_{\varepsilon}^{(1)} \subset S_{\varepsilon, 1}^{+}$satisfying $m_{1}\left(\mathcal{N}_{\varepsilon}^{(1)}\right) \geq 1-C \varepsilon^{n}$.

As for (39), we apply the above reasoning to the partial derivatives of $S_{\varepsilon}(t) v_{o}^{+}$, i.e., we choose $\left\{w_{1}, \ldots, w_{N}\right\}$ in Definition 3.1 as $\left\{\partial \varphi_{N_{1, \varepsilon}} / \partial x_{i}, \ldots, \partial \varphi_{N_{2, \varepsilon}} / \partial x_{i}\right\}$. In this situation the constant $M_{1}$ in Definition 3.1 is bounded above by $C \cdot \varepsilon$, since (A1) implies $\left\|\nabla \psi_{k}\right\|_{L^{\infty}(\Omega)} \leq C \cdot \kappa_{k}^{1 / 2}$; see Section 2.3 Theorem 3.5 furnishes the validity of (39) on a set $\mathcal{N}_{\varepsilon}^{(2)} \subset S_{\varepsilon, 1}^{+}$satisfying $m_{1}\left(\mathcal{N}_{\varepsilon}^{(2)}\right) \geq 1-C \varepsilon^{n}$. For $\mathcal{G}_{\varepsilon, 1}^{+}=\mathcal{N}_{\varepsilon}^{(1)} \cap \mathcal{N}_{\varepsilon}^{(2)}$ we then have both (38) and (39) for all $v_{o}^{+} \in \mathcal{G}_{\varepsilon, 1}^{+}$.

In order to complete the proof we only have to establish (40). Recall that we can write $v_{o}^{+}=\sum_{k=N_{1, \varepsilon}}^{N_{2, \varepsilon}} c_{k} \varphi_{k} \in X_{\varepsilon}^{+}$. Due to the involved boundary conditions for (13), the explicit representation of the semigroup given in (16), and (19), we obtain with 
integration by parts the identity

$$
\left\|\nabla v^{+}(t)\right\|_{L^{2}(\Omega)}^{2}=\left(-\Delta v^{+}(t), v^{+}(t)\right)_{L^{2}(\Omega)}=\sum_{k=N_{1, \varepsilon}}^{N_{2, \varepsilon}} \frac{\kappa_{k}}{1+\kappa_{k}^{2}} \cdot c_{k}^{2} \cdot e^{2 \lambda_{k, \varepsilon} \cdot t} .
$$

According to (20) the eigenvalue $\kappa_{N_{1, \varepsilon}}$ is bounded below by $C \cdot \varepsilon^{-2}$. This furnishes the inequalities $\kappa_{k} /\left(1+\kappa_{k}^{2}\right) \leq \kappa_{k}^{-1} \leq \kappa_{N_{1, \varepsilon}}^{-1} \leq C \cdot \varepsilon^{2}$ for all $k=N_{1, \varepsilon}, \ldots, N_{2, \varepsilon}$, as well as

$$
\left\|\nabla v^{+}(t)\right\|_{L^{2}(\Omega)}^{2} \leq C \cdot \varepsilon^{2} \cdot e^{2 \lambda_{\varepsilon}^{\max } \cdot t} \cdot \sum_{k=N_{1, \varepsilon}}^{N_{2, \varepsilon}} c_{k}^{2}=C \cdot \varepsilon^{2} \cdot e^{2 \lambda_{\varepsilon}^{\max } \cdot t} \cdot\left\|v_{o}^{+}\right\|_{*}^{2} .
$$

Together with (39) the last estimate yields

$$
\begin{aligned}
\left\|\nabla v^{+}(t)\right\|_{L^{4}(\Omega)}^{4} & =\int_{\Omega}\left|\nabla v^{+}(t)\right|^{4} d x \leq\left\|\nabla v^{+}(t)\right\|_{L^{\infty}(\Omega)}^{2} \cdot\left\|\nabla v^{+}(t)\right\|_{L^{2}(\Omega)}^{2} \\
& \leq C \cdot \varepsilon^{4} \cdot|\ln \varepsilon| \cdot e^{4 \lambda_{\varepsilon}^{\max } \cdot t} \cdot\left\|v_{o}^{+}\right\|_{*}^{4} .
\end{aligned}
$$

This completes the proof of (40).

Remark 4.3. Notice that the set $\mathcal{G}_{\varepsilon}^{+}$is obtained from the dominating subspace $X_{\varepsilon}^{+}$ by removing certain cone-shaped regions. It follows easily from the above lemma that these removed parts are small in the following sense. If vol denotes the canonical Lebesgue measure on the finite-dimensional space $X_{\varepsilon}^{+}$and if $B_{R}(0) \subset X^{1 / 2}$ denotes the ball of radius $R$ centered at 0 , then we have for all sufficiently small $\varepsilon>0$

$$
\frac{\operatorname{vol}\left(\mathcal{G}_{\varepsilon}^{+} \cap B_{R}(0)\right)}{\operatorname{vol}\left(X_{\varepsilon}^{+} \cap B_{R}(0)\right)} \geq 1-C \cdot \varepsilon^{\operatorname{dim} \Omega} \quad \text { for all } \quad R>0 .
$$

Therefore, as $\varepsilon \rightarrow 0$ the sets $\mathcal{G}_{\varepsilon}^{+}$cover as large a percentage of the dominating subspace $X_{\varepsilon}^{+}$as we wish.

The following proposition is the main result of this section. It provides sharp bounds on the nonlinearity of the Cahn-Hilliard equation.

Proposition 4.4. Assume that (A1) and (A3) are satisfied, let $F$ be as in (15), and let $\delta^{*}>0$. Consider the dominating subspace $X_{\varepsilon}^{+}$from (8), and let $\mathcal{G}_{\varepsilon}^{+}$denote its subset constructed in Lemma 4.2 for $T=1$. Then there exist constants $C_{1}$ and $C_{2}$ such that for all sufficiently small values of $\varepsilon>0$ the following is true.

Let $v_{o}^{+} \in \mathcal{G}_{\varepsilon}^{+}$be arbitrary, and let $v^{+}(t)=S_{\varepsilon}(t) v_{o}^{+}$denote the solution of (2) starting at $v_{o}^{+}$. Furthermore, for $0<T^{*} \leq 1$ assume that $u:\left[0, T^{*}\right] \rightarrow X^{1 / 2}$ is any continuous function satisfying both

$$
\|u(t)\|_{L^{\infty}(\Omega)} \leq C_{1}
$$

and

$$
\frac{\left\|u(t)-v^{+}(t)\right\|_{*}}{\left\|v^{+}(t)\right\|_{*}} \leq \delta^{*} \cdot \varepsilon^{2} \cdot \sqrt{|\ln \varepsilon|}
$$

for all $t \in\left[0, T^{*}\right]$. Then for all $t \in\left[0, T^{*}\right]$ we have

$$
\|F(u(t))\|_{L^{2}(\Omega)} \leq C_{2} \cdot\left(\varepsilon^{2} \cdot \sqrt{|\ln \varepsilon|}\right)^{\sigma} \cdot e^{(\sigma+1) \cdot \lambda_{\varepsilon}^{\max } \cdot t} \cdot\left\|v_{o}^{+}\right\|_{*}^{\sigma+1} .
$$


Proof. Due to Sobolev's embedding theorem there exists a constant $C$ which depends only on the domain $\Omega$ such that

$$
\|\Upsilon\|_{L^{\infty}(\Omega)} \leq C\|\Upsilon\|_{*} \quad \text { for all } \quad \Upsilon \in H^{2}(\Omega) .
$$

Together with (38), (42), and Lemma 2.1 we therefore obtain for all $t \in\left[0, T^{*}\right]$ the estimate

$$
\begin{aligned}
\|u(t)\|_{L^{\infty}(\Omega)} & \leq C \cdot\left\|u(t)-v^{+}(t)\right\|_{*}+\left\|v^{+}(t)\right\|_{L^{\infty}(\Omega)} \\
& \leq C \cdot \delta^{*} \cdot \varepsilon^{2} \cdot \sqrt{|\ln \varepsilon|} \cdot\left\|v^{+}(t)\right\|_{*}+\left\|v^{+}(t)\right\|_{L^{\infty}(\Omega)} \\
& \leq C \cdot \varepsilon^{2} \cdot \sqrt{|\ln \varepsilon|} \cdot e^{\lambda_{\varepsilon}^{\max } \cdot t} \cdot\left\|v_{o}^{+}\right\|_{*} .
\end{aligned}
$$

According to Adams [1] we also have the Sobolev embedding $H^{2}(\Omega) \hookrightarrow W^{1,4}(\Omega)$, i.e., there exists a constant $C$ which depends only on the domain $\Omega$ such that

$$
\|\nabla \Upsilon\|_{L^{4}(\Omega)} \leq C\|\Upsilon\|_{*} \quad \text { for all } \quad \Upsilon \in H^{2}(\Omega) .
$$

Together with (40), (42), and Lemma 2.1 we now obtain for all $t \in\left[0, T^{*}\right]$ the estimate

$$
\begin{aligned}
\|\nabla u(t)\|_{L^{4}(\Omega)} & \leq C \cdot\left\|u(t)-v^{+}(t)\right\|_{*}+\left\|\nabla v^{+}(t)\right\|_{L^{4}(\Omega)} \\
& \leq C \cdot \delta^{*} \cdot \varepsilon^{2} \cdot \sqrt{|\ln \varepsilon|} \cdot\left\|v^{+}(t)\right\|_{*}+\left\|\nabla v^{+}(t)\right\|_{L^{4}(\Omega)} \\
& \leq C \cdot\left(\varepsilon^{2} \cdot \sqrt{|\ln \varepsilon|}\right)^{1 / 2} \cdot e^{\lambda_{\varepsilon}^{\max } \cdot t} \cdot\left\|v_{o}^{+}\right\|_{*} .
\end{aligned}
$$

Furthermore, (42) furnishes for all $t \in\left[0, T^{*}\right]$ and all sufficiently small $\varepsilon>0$ the estimate

$$
\begin{aligned}
\|u(t)\|_{*} & \leq\left\|u(t)-v^{+}(t)\right\|_{*}+\left\|v^{+}(t)\right\|_{*} \\
& \leq\left(\delta^{*} \cdot \varepsilon^{2} \cdot \sqrt{|\ln \varepsilon|}+1\right) \cdot\left\|v^{+}(t)\right\|_{*} \\
& \leq C \cdot e^{\lambda_{\varepsilon}^{\max } \cdot t} \cdot\left\|v_{o}^{+}\right\|_{*} .
\end{aligned}
$$

Since $\tilde{g}$ is a $C^{2}$-function on an open interval containing 0 there exist constants $C>0$ and $C_{1}>0$ such that

$$
\left|g^{\prime}(s)\right| \leq C \cdot|s|^{\sigma} \quad \text { and } \quad\left|g^{\prime \prime}(s)\right| \leq C \cdot|s|^{\sigma-1} \quad \text { for all } \quad|s| \leq C_{1},
$$

and (41) implies the validity of both

$$
\left\|g^{\prime}(u(t))\right\|_{L^{\infty}(\Omega)} \leq C \cdot\|u(t)\|_{L^{\infty}(\Omega)}^{\sigma} \quad \text { and } \quad\left\|g^{\prime \prime}(u(t))\right\|_{L^{\infty}(\Omega)} \leq C \cdot\|u(t)\|_{L^{\infty}(\Omega)}^{\sigma-1}
$$

on $\left[0, T^{*}\right]$. Together with $F(u)=-g^{\prime}(u) \Delta u-g^{\prime \prime}(u)|\nabla u|^{2}$, estimates (44) and (45)) finally imply for all $t \in\left[0, T^{*}\right]$ the inequality

$$
\begin{aligned}
\|F(u(t))\|_{L^{2}(\Omega) \leq} \leq & \left\|g^{\prime}(u(t))\right\|_{L^{\infty}(\Omega)} \cdot\|\Delta u(t)\|_{L^{2}(\Omega)} \\
& \quad+\left\|g^{\prime \prime}(u(t))\right\|_{L^{\infty}(\Omega)} \cdot\|\nabla u(t)\|_{L^{4}(\Omega)}^{2} \\
\leq \quad & C \cdot\left(\varepsilon^{2} \cdot \sqrt{|\ln \varepsilon|}\right)^{\sigma} \cdot e^{\sigma \cdot \lambda_{\varepsilon}^{\max } \cdot t} \cdot\left\|v_{o}^{+}\right\|_{*}^{\sigma} \cdot\|u(t)\|_{*} \\
& \quad+C \cdot\left(\varepsilon^{2} \cdot \sqrt{|\ln \varepsilon|}\right)^{\sigma} \cdot e^{(\sigma+1) \cdot \lambda_{\varepsilon}^{\max } \cdot t} \cdot\left\|v_{o}^{+}\right\|_{*}^{\sigma+1} .
\end{aligned}
$$

Combining this with (46) furnishes the desired estimate (43). 
If we compare the above proposition with Lemma 4.1 the improvement is evident. The multiplicative constant in the estimate (43) no longer depends on the dimension $n$ of $\Omega$. Especially for $n=3$ the new factor is orders of magnitude smaller than the old one. Notice however that we are forced to obtain the estimate for complete solution pieces, due to the nature of the results in Section 3 . If we would only prove an exact analogue to Lemma 4.1, it would be far from obvious whether for arbitrary $t>0$ the function $v^{+}(t)$ still satisfies the sharp $L^{\infty}(\Omega)$-bounds.

The functional-analytic setting described in Section 2 showed that we study the semiflow of the nonlinear Cahn-Hilliard equation (13) in the Hilbert space $X^{1 / 2}$. Thus, we need to be able to deduce (41) in Proposition 4.4 from knowledge of the size of $u(t)$ with respect to the norm $\|\cdot\|_{*}$. It was mentioned in the above proof that Sobolev's embedding theorem implies $\|u(t)\|_{L^{\infty}(\Omega)} \leq C \cdot\|u(t)\|_{*}$. Therefore, (41) is satisfied as long as $\|u(t)\|_{*} \leq C_{1} / C$ for all $t \in\left[0, T^{*}\right]$. Unfortunately, this estimate is not sufficient for our applications. Rather we have to employ the following lemma, which relaxes the necessary condition on $\|u(t)\|_{*}$ considerably.

Lemma 4.5. Assume that (A1) is satisfied, and let $C_{1}>0$ and $\delta^{*} \in(0,1)$ be arbitrary. Consider the dominating subspace $X_{\varepsilon}^{+}$from (8) for some $\gamma_{o} \in(0,1)$, and let $\mathcal{G}_{\varepsilon}^{+}$denote its subset constructed in Lemma 4.2 for $T=1$. Then there exists a constant $M$ such that for all sufficiently small values of $\varepsilon>0$ the following is true.

Let $v_{o}^{+} \in \mathcal{G}_{\varepsilon}^{+}$be arbitrary, and let $v^{+}(t)=S_{\varepsilon}(t) v_{o}^{+}$denote the solution of (2) starting at $v_{o}^{+}$. Furthermore, for $0<T^{*} \leq 1$ assume that $u:\left[0, T^{*}\right] \rightarrow X^{1 / 2}$ is any continuous function such that for all $t \in\left[0, T^{*}\right]$ both

$$
\|u(t)\|_{*} \leq M \cdot\left(\varepsilon^{2} \cdot \sqrt{|\ln \varepsilon|}\right)^{-\gamma_{o}} \cdot\left\|v_{o}^{+}\right\|_{*}^{1-\gamma_{o}}
$$

and

$$
\frac{\left\|u(t)-v^{+}(t)\right\|_{*}}{\left\|v^{+}(t)\right\|_{*}} \leq \delta^{*} \cdot \varepsilon^{2} \cdot \sqrt{|\ln \varepsilon|}
$$

hold. Then the estimate (43) is satisfied on $\left[0, T^{*}\right]$.

Proof. We can assume without loss of generality that $\varepsilon<1$, and one can easily verify that

$$
\varepsilon^{2} \cdot \sqrt{|\ln \varepsilon|} \leq \frac{1}{2 \cdot \sqrt{e}}<\frac{1}{2} \quad \text { for all } \quad 0<\varepsilon<1 .
$$

Together with (48) and $\delta^{*} \in(0,1)$ this furnishes

$$
\left\|v^{+}\left(T^{*}\right)\right\|_{*} \leq \delta^{*} \cdot \varepsilon^{2} \cdot \sqrt{|\ln \varepsilon|} \cdot\left\|v^{+}\left(T^{*}\right)\right\|_{*}+\left\|u\left(T^{*}\right)\right\|_{*} \leq \frac{\left\|v^{+}\left(T^{*}\right)\right\|_{*}}{2}+\left\|u\left(T^{*}\right)\right\|_{*},
$$

and therefore $\left\|v^{+}\left(T^{*}\right)\right\|_{*} \leq 2 \cdot\left\|u\left(T^{*}\right)\right\|_{*}$. Combining this estimate with (47) and the lower bound on the growth of $\left\|v^{+}(t)\right\|_{*}$ provided by Lemma 2.1 yields

$$
\left\|v_{o}^{+}\right\|_{*} \cdot e^{\gamma_{o} \cdot \lambda_{\varepsilon}^{\max } \cdot T^{*}} \leq\left\|v^{+}\left(T^{*}\right)\right\|_{*} \leq 2 M \cdot\left(\varepsilon^{2} \cdot \sqrt{|\ln \varepsilon|}\right)^{-\gamma_{o}} \cdot\left\|v_{o}^{+}\right\|_{*}^{1-\gamma_{o}},
$$

as well as

$$
e^{\lambda_{\varepsilon}^{\max } \cdot T^{*}} \leq(2 M)^{1 / \gamma_{o}} \cdot\left(\varepsilon^{2} \cdot \sqrt{|\ln \varepsilon|} \cdot\left\|v_{o}^{+}\right\|_{*}\right)^{-1} .
$$


Finally, this estimate, together with (44) from Proposition 4.4 (which is still valid under the assumptions of this lemma), furnishes for all $t \in\left[0, T^{*}\right]$ the inequality

$$
\|u(t)\|_{L^{\infty}(\Omega)} \leq C \cdot \varepsilon^{2} \cdot \sqrt{|\ln \varepsilon|} \cdot\left\|v_{o}^{+}\right\|_{*} \cdot e^{\lambda_{\varepsilon}^{\max } \cdot T^{*}} \leq C \cdot(2 M)^{1 / \gamma_{o}},
$$

and with $M=\left(C_{1} / C\right)^{\gamma_{o}} / 2$ the lemma follows.

4.2. The later stages of spinodal decomposition. In this section we improve the main result of Sander and Wanner [34, which was stated without any technical details in Theorem 1.1. One of the omitted details concerned the precise notion of closeness of $u_{o}$ to the dominating subspace $X_{\varepsilon}^{+}$. In order for Theorem 1.1 to hold, the initial condition $u_{o}$ has to be contained in a cone $\mathcal{K}_{\varepsilon, \delta_{\varepsilon}}$ as in (35), where $\delta_{\varepsilon}=\delta_{o} \cdot \varepsilon^{2-\operatorname{dim} \Omega / 2}$ for some fixed $\delta_{o} \in(0,1 / 2)$. Only then is it possible to apply Lemma 4.1, which lies at the heart of proving Theorem 1.1.

The family of examples $w_{\varepsilon}$ constructed in Section 4.1 showed that any improvement of the crucial estimates in 34 has to be accompanied by a further restriction on the initial conditions $u_{o}$. For this, we introduce the following definition.

Definition 4.6. Let $X_{\varepsilon}^{+}$denote the dominating subspace defined in (8), let $X_{\varepsilon}^{-}$ denote its orthogonal complement in the Hilbert space $X^{1 / 2}$, and let $\mathcal{G}_{\varepsilon}^{+}$denote the set from Lemma 4.2 for $T=1$. Then for every sufficiently small $\varepsilon>0$ and for every $\delta>0$ we define

$$
\mathcal{G}_{\varepsilon, \delta}=\left\{u=u^{+}+u^{-} \in X_{\varepsilon}^{+} \oplus X_{\varepsilon}^{-} \quad: u \in \mathcal{K}_{\varepsilon, \delta} \quad \text { and } \quad u^{+} \in \mathcal{G}_{\varepsilon}^{+}\right\},
$$

where $\mathcal{K}_{\varepsilon, \delta}$ was defined in (35).

The sets $\mathcal{G}_{\varepsilon, \delta}$ consist of all functions in the cone $\mathcal{K}_{\varepsilon, \delta}$ whose orthogonal projection onto the dominating subspace is contained in the set $\mathcal{G}_{\varepsilon}^{+}$. They constitute the collection of all "good" initial conditions $u_{o}$, i.e., of all initial conditions for which we can improve Theorem 1.1 Recall that according to Remark 4.3 in a measuretheoretic sense the set $\mathcal{G}_{\varepsilon}^{+}$is a large subset of the dominating subspace $X_{\varepsilon}^{+}$.

Definition 4.6. Lemma 2.1. and Proposition 4.4 are all we need to obtain our main result. The proof is based on similar results by Sander and Wanner [34]. In order to keep the presentation as simple as possible, we formulate the result directly for the Cahn-Hilliard equation. It can easily be adapted to the more general case of evolution equations in $X^{\alpha}$-spaces. This will be indicated briefly in the last section.

Theorem 4.7. Consider the Cahn-Hilliard equation (13). Furthermore, assume that both (A1) and (A3) are satisfied, and adopt the notation introduced in Definition 4.6. Finally, choose and fix constants $c>0, \delta_{o} \in(0,1 / 3)$ and $\varrho \in(0,1)$.

Then there exist $\varepsilon$-independent constants $D>0$ and $\gamma_{o} \in(0,1)$ (the latter determines the dominating subspace $X_{\varepsilon}^{+}$) such that for all sufficiently small $\varepsilon>0$ the following holds. If $u_{o} \in \mathcal{G}_{\varepsilon, \delta_{\varepsilon}}$ with $\delta_{\varepsilon}=\delta_{o} \cdot \varepsilon^{2} \cdot \sqrt{|\ln \varepsilon|}$ is any initial condition satisfying

$$
e^{-c / \varepsilon} \leq\left\|u_{o}\right\|_{*} \leq \min \left\{1, \quad\left(D \cdot \varepsilon^{-2 \cdot(1-1 / \sigma)+\varrho}\right)^{1 /(1-\varrho)}\right\},
$$

and if $u$ and $v$ denote the solutions of equations (13) and (21), respectively, starting at $u_{o}$, then there exists a first time $T_{o}>0$ such that

$$
\left\|u\left(T_{o}\right)\right\|_{*}=D \cdot \varepsilon^{-2 \cdot(1-1 / \sigma)+\varrho} \cdot\left\|u_{o}\right\|_{*}^{\varrho},
$$


and for all $t \in\left[0, T_{o}\right]$ we have

$$
\frac{\|u(t)-v(t)\|_{*}}{\|v(t)\|_{*}} \leq \delta_{\varepsilon}=\delta_{o} \cdot \varepsilon^{2} \cdot \sqrt{|\ln \varepsilon|} .
$$

Proof. Choose a constant $0<\gamma_{o}<1$ such that both

$$
\frac{(\sigma+1)\left(1-\gamma_{o}\right)}{\sigma+1-\gamma_{o}}<\varrho \quad \text { and } \quad \frac{-2 \gamma_{o} \cdot(\sigma-1)}{\sigma+1-\gamma_{o}}<-2 \cdot\left(1-\frac{1}{\sigma}\right)+\varrho
$$

are satisfied. The second of these estimates allows us to choose a constant $D_{1}>0$ such that for all sufficiently small $\varepsilon>0$ we have

$$
D_{1} \cdot \varepsilon^{-2 \cdot(1-1 / \sigma)+\varrho} \leq\left(\varepsilon^{-2} \cdot|\ln \varepsilon|^{-1 / 2}\right)^{\gamma_{o} \cdot(\sigma-1) /\left(\sigma+1-\gamma_{o}\right)},
$$

since the function $\varepsilon^{-2 \cdot(1-1 / \sigma)+\varrho+2 \gamma_{o} \cdot(\sigma-1) /\left(\sigma+1-\gamma_{o}\right)} \cdot|\ln \varepsilon|^{(1 / 2) \cdot \gamma_{o} \cdot(\sigma-1) /\left(\sigma+1-\gamma_{o}\right)}$ converges to 0 as $\varepsilon \rightarrow 0$. The constant $D$ appearing in the formulation of the theorem is defined as $D=D_{1} \cdot D_{2}$, where the precise choice of $D_{2}>0$ will be made later in the proof.

Let $\gamma_{\varepsilon}=\gamma_{o} \cdot \lambda_{\varepsilon}^{\max }$, and adopt the notation of Lemma 2.1. Choose an initial condition $u_{o}$ as in the formulation of the theorem, let $u_{o}=u_{o}^{+}+u_{o}^{-} \in X_{\varepsilon}^{+} \oplus X_{\varepsilon}^{-}$, and define $v_{o}^{+}=u_{o}^{+}$. Then the orthogonal projection of the solution $v(t)$ onto $X_{\varepsilon}^{+}$is given by $v^{+}(t)=S_{\varepsilon}(t) v_{o}^{+}$. Furthermore, according to our choice of $u_{o}$ we have $v_{o}^{+} \in$ $\mathcal{G}_{\varepsilon}^{+}$, as well as

$$
\frac{\left\|u(0)-v^{+}(0)\right\|_{*}}{\left\|v^{+}(0)\right\|_{*}}=\frac{\left\|u_{o}-v_{o}^{+}\right\|_{*}}{\left\|v_{o}^{+}\right\|_{*}}=\frac{\left\|u_{o}^{-}\right\|_{*}}{\left\|u_{o}^{+}\right\|_{*}} \leq \frac{\delta^{*}}{3} \cdot \varepsilon^{2} \cdot \sqrt{|\ln \varepsilon|},
$$

if we define $\delta^{*}=3 \delta_{o}<1$. Due to this estimate and the continuity of $u$ and $v^{+}$, we can choose $T^{*} \in(0,1]$ maximal such that for all $t \in\left[0, T^{*}\right]$ we have

$$
\frac{\left\|u(t)-v^{+}(t)\right\|_{*}}{\left\|v^{+}(t)\right\|_{*}} \leq \delta^{*} \cdot \varepsilon^{2} \cdot \sqrt{|\ln \varepsilon|},
$$

as well as

$$
\|u(t)\|_{*} \leq D \cdot \varepsilon^{-2 \cdot(1-1 / \sigma)+\varrho} \cdot\left\|u_{o}\right\|_{*}^{\varrho},
$$

where $D=D_{1} \cdot D_{2}$. (Notice that according to (50), the right-hand side of 56) is strictly larger than $\left\|u_{o}\right\|_{*}$.) It is straightforward to verify that for $D_{2} \leq M / 2$, where $M$ denotes the constant from Lemma 4.5) our definition of $D$ and (154) imply

$$
\begin{aligned}
D \cdot \varepsilon^{-2 \cdot(1-1 / \sigma)+\varrho} & \leq D_{2} \cdot\left(\varepsilon^{-2} \cdot|\ln \varepsilon|^{-1 / 2}\right)^{\gamma_{o} \cdot(\sigma-1) /\left(\sigma+1-\gamma_{o}\right)} \\
& \leq \frac{M}{2} \cdot\left(\varepsilon^{-2} \cdot|\ln \varepsilon|^{-1 / 2}\right)^{\gamma_{o}}
\end{aligned}
$$

as long as $\varepsilon$ is sufficiently small; in fact, $\varepsilon<1$ suffices due to (49). The choice of $u_{o}$ furnishes with (49) and $\delta_{o}<1 / 3$

$$
\left\|u_{o}\right\|_{*}=\sqrt{\left\|u_{o}^{+}\right\|_{*}^{2}+\left\|u_{o}^{-}\right\|_{*}^{2}} \leq \sqrt{1+\delta_{o}^{2} \cdot \varepsilon^{4} \cdot|\ln \varepsilon|} \cdot\left\|u_{o}^{+}\right\|_{*} \leq 2 \cdot\left\|u_{o}^{+}\right\|_{*} .
$$

Combined with $u_{o}^{+}=v_{o}^{+}$, (50), and (53), this estimate yields

$$
\left\|u_{o}\right\|_{*}^{\varrho} \leq 2 \cdot\left\|v_{o}^{+}\right\|_{*}^{\varrho} \leq 2 \cdot\left\|v_{o}^{+}\right\|_{*}^{(\sigma+1) \cdot\left(1-\gamma_{o}\right) /\left(\sigma+1-\gamma_{o}\right)} \leq 2 \cdot\left\|v_{o}^{+}\right\|_{*}^{1-\gamma_{o}} .
$$

Together, these estimates show that the validity of (56) on $\left[0, T^{*}\right]$ implies the validity of (47). Thus, Lemma 4.5 furnishes that all the assumptions of Proposition 4.4 are 
satisfied on $\left[0, T^{*}\right]$, and we can use its nonlinearity estimate. Due to the definition of $u$ and $v$, the variation of constants formula gives

$$
u(t)-v(t)=\int_{0}^{t} S_{\varepsilon}(t-s) F(u(s)) d s,
$$

and Lemma 2.1 (with any $\beta_{o} \in(0,1)$ ) and Proposition 4.4 imply for all $t \in\left[0, T^{*}\right]$ the estimate

$$
\begin{aligned}
&\|u(t)-v(t)\|_{*} \leq \int_{0}^{t} K_{\varepsilon} \cdot(t-s)^{-1 / 2} \cdot e^{\beta_{\varepsilon} \cdot(t-s)} \cdot\|F(u(s))\|_{L^{2}(\Omega)} d s \\
& \leq K_{\varepsilon} \cdot C_{2} \cdot \varepsilon^{2 \sigma} \cdot|\ln \varepsilon|^{\sigma / 2} \cdot\left\|v_{o}^{+}\right\|_{*}^{\sigma+1} \cdot e^{(\sigma+1) \cdot \lambda_{\varepsilon}^{\max } \cdot t} \\
& \cdot \int_{0}^{t}(t-s)^{-1 / 2} \cdot e^{\left(\beta_{\varepsilon}-(\sigma+1) \cdot \lambda_{\varepsilon}^{\max }\right) \cdot(t-s)} d s .
\end{aligned}
$$

The last integral can be bounded further by

$$
\begin{aligned}
& \int_{0}^{t}(t-s)^{-1 / 2} \cdot e^{\left(\beta_{\varepsilon}-(\sigma+1) \cdot \lambda_{\varepsilon}^{\max }\right) \cdot(t-s)} d s=\int_{0}^{t} \tau^{-1 / 2} \cdot e^{-\left(\sigma-\beta_{o}\right) \cdot \lambda_{\varepsilon}^{\max } \cdot \tau} d \tau \\
= & \left(\left(\sigma-\beta_{o}\right) \cdot \lambda_{\varepsilon}^{\max }\right)^{-1 / 2} \cdot \int_{0}^{\left(\sigma-\beta_{o}\right) \cdot \lambda_{\varepsilon}^{\max } \cdot t} s^{-1 / 2} \cdot e^{-s} d s \\
\leq & \left(\left(\sigma-\beta_{o}\right) \cdot \lambda_{\varepsilon}^{\max }\right)^{-1 / 2} \cdot \int_{0}^{\infty} s^{-1 / 2} \cdot e^{-s} d s .
\end{aligned}
$$

Combining the last two estimates with Lemma 2.1 and (6) furnishes an $\varepsilon$-independent constant $C_{o}>0$ such that for all $t \in\left[0, T^{*}\right]$ we have

$$
\|u(t)-v(t)\|_{*} \leq C_{o} \cdot \varepsilon^{2 \sigma} \cdot|\ln \varepsilon|^{\sigma / 2} \cdot\left\|v_{o}^{+}\right\|_{*}^{\sigma+1} \cdot e^{(\sigma+1) \cdot \lambda_{\varepsilon}^{\max } \cdot t} .
$$

According to Lemma 2.1 we have $\left\|v^{+}(t)\right\|_{*} \geq e^{\gamma_{\varepsilon} \cdot t} \cdot\left\|v_{o}^{+}\right\|_{*}$, and with $\gamma_{\varepsilon}=\gamma_{o} \cdot \lambda_{\varepsilon}^{\max }$ we finally obtain

$$
\frac{\|u(t)-v(t)\|_{*}}{\left\|v^{+}(t)\right\|_{*}} \leq C_{o} \cdot \varepsilon^{2 \sigma} \cdot|\ln \varepsilon|^{\sigma / 2} \cdot\left\|v_{o}^{+}\right\|_{*}^{\sigma} \cdot e^{\left(\sigma+1-\gamma_{o}\right) \cdot \lambda_{\varepsilon}^{\max } \cdot t} .
$$

Furthermore, for all $t \in\left[0, T^{*}\right]$ Lemma 2.1$]$ implies

$$
\frac{\left\|v(t)-v^{+}(t)\right\|_{*}}{\left\|v^{+}(t)\right\|_{*}}=\frac{\left\|S_{\varepsilon}(t)\left(u_{o}-v_{o}^{+}\right)\right\|_{*}}{\left\|S_{\varepsilon}(t) v_{o}^{+}\right\|_{*}} \leq \frac{\left\|u_{o}-v_{o}^{+}\right\|_{*}}{\left\|v_{o}^{+}\right\|_{*}} \leq \frac{\delta^{*}}{3} \cdot \varepsilon^{2} \cdot \sqrt{|\ln \varepsilon|},
$$

since $u_{o}-v_{o}^{+}=u_{o}^{-} \in X_{\varepsilon}^{-}$, and therefore $\left\|S_{\varepsilon}(t)\left(u_{o}-v_{o}^{+}\right)\right\|_{*} \leq e^{\gamma_{\varepsilon} \cdot t} \cdot\left\|u_{o}-v_{o}^{+}\right\|_{*}$.

The final step of the proof consists in deriving an upper bound on the time $T^{*}$, so that we can bound the relative distance given in (58). To this end, (49), (55), and $\delta^{*} \in(0,1)$ imply

$$
\left\|v^{+}\left(T^{*}\right)\right\|_{*} \leq \delta^{*} \cdot \varepsilon^{2} \cdot \sqrt{|\ln \varepsilon|} \cdot\left\|v^{+}\left(T^{*}\right)\right\|_{*}+\left\|u\left(T^{*}\right)\right\|_{*} \leq \frac{\left\|v^{+}\left(T^{*}\right)\right\|_{*}}{2}+\left\|u\left(T^{*}\right)\right\|_{*},
$$

and therefore $\left\|v^{+}\left(T^{*}\right)\right\|_{*} \leq 2 \cdot\left\|u\left(T^{*}\right)\right\|_{*}$. Together with (53), (54), (56), (57), and Lemma 2.1 this furnishes

$$
\begin{aligned}
\left\|v_{o}^{+}\right\|_{*} \cdot e^{\gamma_{o} \cdot \lambda_{\varepsilon}^{\max } \cdot T^{*}} \leq & \left\|v^{+}\left(T^{*}\right)\right\|_{*} \leq 4 D_{2} \cdot D_{1} \varepsilon^{-2 \cdot(1-1 / \sigma)+\varrho} \cdot\left\|v_{o}^{+}\right\|_{*}^{\varrho} \\
\leq & 4 D_{2} \cdot\left(\varepsilon^{2}|\ln \varepsilon|^{1 / 2}\right)^{\gamma_{o} \cdot(1-\sigma) /\left(\sigma+1-\gamma_{o}\right)} \\
& \cdot\left\|v_{o}^{+}\right\|_{*}^{(\sigma+1)\left(1-\gamma_{o}\right) /\left(\sigma+1-\gamma_{o}\right)}
\end{aligned}
$$


and therefore

$$
e^{\lambda_{\varepsilon}^{\max } \cdot T^{*}} \leq\left(4 D_{2}\right)^{1 / \gamma_{o}} \cdot\left(\varepsilon^{2}|\ln \varepsilon|^{1 / 2}\right)^{(1-\sigma) /\left(\sigma+1-\gamma_{o}\right)} \cdot\left\|v_{o}^{+}\right\|_{*}^{-\sigma /\left(\sigma+1-\gamma_{o}\right)} .
$$

This inequality constitutes our desired upper bound for $T^{*}$, and together with (58) it furnishes for all $t \in\left[0, T^{*}\right]$ the estimate

$$
\frac{\|u(t)-v(t)\|_{*}}{\left\|v^{+}(t)\right\|_{*}} \leq C_{o} \cdot\left(4 D_{2}\right)^{\left(\sigma+1-\gamma_{o}\right) / \gamma_{o}} \cdot \varepsilon^{2} \cdot \sqrt{|\ln \varepsilon|} .
$$

Finally, we can choose our constant $D_{2}$. According to the paragraph after (566) we need at least $D_{2} \leq M / 2$. Assume in addition that $C_{o} \cdot\left(4 D_{2}\right)^{\left(\sigma+1-\gamma_{o}\right) / \gamma_{o}} \leq \delta^{*} / 3$ is satisfied. Then the above estimate and (59) yield both

$$
\frac{\|u(t)-v(t)\|_{*}}{\|v(t)\|_{*}} \leq \frac{\|u(t)-v(t)\|_{*}}{\left\|v^{+}(t)\right\|_{*}} \leq \frac{\delta^{*}}{3} \cdot \varepsilon^{2} \cdot \sqrt{|\ln \varepsilon|}=\delta_{o} \cdot \varepsilon^{2} \cdot \sqrt{|\ln \varepsilon|}
$$

and

$$
\frac{\left\|u(t)-v^{+}(t)\right\|_{*}}{\left\|v^{+}(t)\right\|_{*}} \leq \frac{2}{3} \cdot \delta^{*} \cdot \varepsilon^{2} \cdot \sqrt{|\ln \varepsilon|}
$$

for all $t \in\left[0, T^{*}\right]$. On the one hand, this shows that (52) holds everywhere on $\left[0, T^{*}\right]$. On the other hand, since we chose $T^{*} \in(0,1]$ maximal such that both (55) and (56) are valid on $\left[0, T^{*}\right]$, we necessarily have to have $\left\|u\left(T^{*}\right)\right\|_{*}=D \cdot \varepsilon^{-2 \cdot(1-1 / \sigma)+\varrho} \cdot\left\|u_{o}\right\|_{*}^{\varrho}$, unless $T^{*}=1$. Notice however that (60) and the assumed lower bound on $\left\|u_{o}\right\|_{*}$ furnish $T^{*}=O(\varepsilon)$ for $\varepsilon \rightarrow 0$, i.e., $T^{*}<1$ as long as $\varepsilon>0$ is sufficiently small and the theorem finally follows with $T_{o}=T^{*}$.

Remark 4.8. We would like to point out that the assumed lower bound on the norm of the initial condition $u_{o}$ could easily be relaxed. In Lemma 4.2 it is possible to allow for a maximal time $T$ which is polynomial in $\varepsilon^{-1}$, without changing any of the results. This is however not necessary for our application, since initial conditions which are exponentially close to the homogeneous equilibrium are not physically reasonable. See also the discussion in [28].

The above theorem is best possible in the sense that the size of $\left\|u\left(T_{o}\right)\right\|_{*}$ given in (51) exhibits the correct $\varepsilon$-scaling for randomly chosen initial conditions. For example, for the case of the classical Cahn-Hilliard nonlinearity $f(u)=u-u^{3}$ and total mass $\mu=0$, the theorem predicts an order of roughly $\varepsilon^{-1+\varrho}$, where $\varrho>0$ is arbitrarily small. Once the norm of $u$ reaches this threshold, the relative distance between $u$ and $v$ exceeds $O\left(\varepsilon^{2} \sqrt{|\ln \varepsilon|}\right)$. This was confirmed numerically in the right diagram of Figure 2. We performed similar simulations for other nonlinearities, as well as for other values of $\mu$ or $\sigma$. The results consistently reproduce the order predicted by Theorem 4.7 .

From a practical point of view, one is of course interested in obtaining lower bounds on the $L^{\infty}(\Omega)$-norm of the solution $u$, up to which linear behavior determines the pattern selection. It was shown by Maier-Paape and Wanner in [29. Remark 3.6] that for any function $v^{+} \in X_{\varepsilon}^{+}$we have $\left\|v^{+}\right\|_{L^{\infty}(\Omega)} \geq C \cdot \varepsilon^{2} \cdot\left\|v^{+}\right\|_{*}$ for some $\varepsilon$-independent positive constant $C$. According to the proof of Theorem 4.7 the sizes of both $v^{+}(t)$ and $u(t)$ remain of the same order on the whole interval $\left[0, T_{o}\right]$, and therefore we obtain $\left\|v^{+}\left(T_{o}\right)\right\|_{L^{\infty}(\Omega)} \geq C \cdot \varepsilon^{2 / \sigma+\varrho} \cdot\left\|u_{o}\right\|_{*}$. Combining this with (52), one can show that we also have

$$
\left\|u\left(T_{o}\right)\right\|_{L^{\infty}(\Omega)} \geq C \cdot \varepsilon^{2 / \sigma+\varrho} \cdot\left\|u_{o}\right\|_{*} .
$$


In other words, for the classical Cahn-Hilliard nonlinearity $f(u)=u-u^{3}$ and total mass $\mu=0$, the theorem predicts an $L^{\infty}(\Omega)$-order of roughly $\varepsilon^{1+\varrho}$, where $\varrho>0$ is arbitrarily small. Furthermore, by choosing suitable nonlinearities, we can push this order as close as we want to an $\varepsilon$-independent constant.

\section{Conclusions}

The results of this paper explain spinodal decomposition up to distances from the homogeneous equilibrium which considerably improve previous results due to Maier-Paape and Wanner [28, 29] and Sander and Wanner [34. The level of improvement increases with the dimension of the domain, and we end up with dimension-independent results for the first time. Moreover, our results manage to close the gap between analytical methods and numerical simulations by explaining why the optimal results of [34] are not observed in practice.

Despite establishing optimal results, several open questions remain. Ultimately, one is interested in obtaining an estimate for the size of $u$ when the relative distance between $u$ and $v$ reaches an $\varepsilon$-independent small threshold. Numerical simulations indicate an order of $\varepsilon^{-2}$ in both one and two space dimensions. Based on the results of this paper we expect the same order in three dimensions as well. While we can basically achieve this order for specific nonlinearities, the classic case remains open. We are confident that it is possible to extend our methods to also cover these last stages of the decomposition process, by allowing for larger relative distances in Proposition 4.4. This would of course yield weaker forms of the estimate (43), but an iterative application of the methods of Theorem 4.7 will allow us to employ these towards extending the maximal radius. In addition, it is not immediately clear that most solutions originating near the homogeneous equilibrium end up in the set $\mathcal{G}_{\varepsilon, \delta_{\varepsilon}}$ at the end of the first decomposition stage studied in [28, 29]. Also this question can probably be settled by the iterative application of our main techniques mentioned before. These results will be presented elsewhere.

Although we presented our results only for the case of spinodal decomposition in the Cahn-Hilliard equation, applications to other transient pattern formation phenomena are possible. Garcke, Maier-Paape, and Weikard 21] recently considered spinodal decomposition in the presence of elasticity by adapting the results of [28, 29]. In their situation, the linearization of the problem changes, but the nonlinearity remains basically unchanged - allowing for a direct application of our results. In addition, our results can be carried over to the situation of pattern formation in parabolic systems caused by Turing instabilities [19, 31, 36]. In a recent paper, Sander and Wanner [35] demonstrated that the results of [28, 29, 34] can be adapted to this new situation - and using their techniques it is possible to apply the results of this paper as well. This will also be presented elsewhere.

\section{REFERENCES}

[1] R. A. Adams. Sobolev Spaces. Academic Press, San Diego - London, 1975. MR 56:9247

[2] R. Aurich, A. Bäcker, R. Schubert, and M. Taglieber. Maximum norms of chaotic quantum eigenstates and random waves. Physica D, 129:1-14, 1999. MR 2000d:81042

[3] F. Bai, C. M. Elliott, A. Gardiner, A. Spence, and A. M. Stuart. The viscous Cahn-Hilliard equation. Part I: Computations. Nonlinearity, 8:131-160, 1995. MR 95m:35082

[4] F. Bai, A. Spence, and A. M. Stuart. Numerical computations of coarsening in the onedimensional Cahn-Hilliard model of phase separation. Physica D, 78:155-165, 1994. MR 95g:65136 
[5] H. Bauer. Probability Theory. de Gruyter, Berlin, 1996. MR 97f:60001

[6] J. F. Blowey and C. M. Elliott. The Cahn-Hilliard gradient theory for phase separation with nonsmooth free energy. I. Mathematical analysis. European Journal of Applied Mathematics, 2:233-280, 1991. MR 93a:35025

[7] J. F. Blowey and C. M. Elliott. The Cahn-Hilliard gradient theory for phase separation with nonsmooth free energy. II. Numerical analysis. European Journal of Applied Mathematics, 3:147-179, 1992. MR 93g:80007

[8] J. W. Cahn. Free energy of a nonuniform system. II. Thermodynamic basis. Journal of Chemical Physics, 30:1121-1124, 1959.

[9] J. W. Cahn. Phase separation by spinodal decomposition in isotropic systems. Journal of Chemical Physics, 42:93-99, 1965.

[10] J. W. Cahn. Spinodal decomposition. Transactions of the Metallurgical Society of AIME, 242:166-180, 1968.

[11] J. W. Cahn and J. E. Hilliard. Free energy of a nonuniform system I. Interfacial free energy. Journal of Chemical Physics, 28:258-267, 1958.

[12] D. L. Cohn. Measure Theory. Birkhäuser, Boston - Basel - Stuttgart, 1980. MR 81k:28001

[13] M. I. M. Copetti. Numerical analysis of nonlinear equations arising in phase transition and thermoelasticity. Ph.D. thesis, University of Sussex, 1991.

[14] M. I. M. Copetti and C. M. Elliott. Kinetics of phase decomposition processes: Numerical solutions to Cahn-Hilliard equation. Materials Science and Technology, 6:273-283, 1990.

[15] K. R. Elder and R. C. Desai. Role of nonlinearities in off-critical quenches as described by the Cahn-Hilliard model of phase separation. Physical Review B, 40:243-254, 1989.

[16] K. R. Elder, T. M. Rogers, and R. C. Desai. Early stages of spinodal decomposition for the Cahn-Hilliard-Cook model of phase separation. Physical Review B, 38:4725-4739, 1988.

[17] C. M. Elliott. The Cahn-Hilliard model for the kinetics of phase separation. In J. F. Rodrigues, editor, Mathematical Models for Phase Change Problems, pages 35-73. Birkhäuser, Basel, 1989. MR 91c: 80014

[18] C. M. Elliott and D. A. French. Numerical studies of the Cahn-Hilliard equation for phase separation. IMA Journal of Applied Mathematics, 38:97-128, 1987. MR 90f:80004

[19] I. R. Epstein and J. A. Pojman. An Introduction to Nonlinear Chemical Dynamics: Oscillations, Waves, Patterns, and Chaos. Oxford University Press, Oxford - New York, 1998.

[20] P. C. Fife. Models for phase separation and their mathematics. Electronic Journal of Differential Equations, 2000(48):1-26, 2000. MR 2001k:35147

[21] H. Garcke, S. Maier-Paape, and U. Weikard. Spinodal decomposition in the presence of elastic interactions. In S. Hildebrandt and H. Karcher, editors, Geometric Analysis and Nonlinear Partial Differential Equations, pages 603-635. Springer-Verlag, Berlin, 2002.

[22] C. P. Grant. Spinodal decomposition for the Cahn-Hilliard equation. Communications in Partial Differential Equations, 18:453-490, 1993. MR 94b:35147.

[23] D. Henry. Geometric Theory of Semilinear Parabolic Equations, volume 840 of Lecture Notes in Mathematics. Springer-Verlag, Berlin - Heidelberg - New York, 1981. MR 83j:35084

[24] J. E. Hilliard. Spinodal decomposition. In H. I. Aaronson, editor, Phase Transformations, pages 497-560. American Society for Metals, Metals Park, Ohio, 1970.

[25] J. M. Hyde, M. K. Miller, M. G. Hetherington, A. Cerezo, G. D. W. Smith, and C. M. Elliott. Spinodal decomposition in Fe-Cr alloys: Experimental study at the atomic level and comparison with computer models. Acta metallurgica et materialia, 43:3385-3426, 1995.

[26] J.-P. Kahane. Some Random Series of Functions. Cambridge University Press, Cambridge London - New York, second edition, 1985. MR 87m:60119

[27] J. S. Langer. Theory of spinodal decomposition in alloys. Annals of Physics, 65:53-86, 1971.

[28] S. Maier-Paape and T. Wanner. Spinodal decomposition for the Cahn-Hilliard equation in higher dimensions. Part I: Probability and wavelength estimate. Communications in Mathematical Physics, 195(2):435-464, 1998. MR 99h:35111

[29] S. Maier-Paape and T. Wanner. Spinodal decomposition for the Cahn-Hilliard equation in higher dimensions: Nonlinear dynamics. Archive for Rational Mechanics and Analysis, 151(3):187-219, 2000. MR 2001d:35083

[30] R. J. Muirhead. Aspects of Multivariate Statistical Theory. Wiley, New York, 1982. MR 84c:62073

[31] J. D. Murray. Mathematical Biology. Springer-Verlag, Berlin, second edition, 1993. MR 94j:92002 
[32] E.-M. Nash. Finite-Elemente und Spektral-Galerkin Verfahren zur numerischen Lösung der Cahn-Hilliard Gleichung und verwandter nichtlinearer Evolutionsgleichungen. Ph.D. thesis, Universität Augsburg, 2000.

[33] E. Sander and T. Wanner. Monte Carlo simulations for spinodal decomposition. Journal of Statistical Physics, 95(5-6):925-948, 1999.

[34] E. Sander and T. Wanner. Unexpectedly linear behavior for the Cahn-Hilliard equation. SIAM Journal on Applied Mathematics, 60(6):2182-2202, 2000. MR 2001i:35161

[35] E. Sander and T. Wanner. Pattern formation in a nonlinear model for animal coats. Journal of Differential Equations, 191(1):143-174, 2003.

[36] A. M. Turing. The chemical basis of morphogenesis. Philosophical Transactions of the Royal Society of London, 237B:37-72, 1952.

Department of Mathematical Sciences, George Mason University, Fairfax, Virginia 22030

E-mail address: wanner@math.gmu.edu 